\title{
NORMS OF MODES AND QUASI-MODES REVISITED
}

\author{
JOHN A. TOTH AND STEVE ZELDITCH
}

\begin{abstract}
This article is devoted to the analysis of eigenfunctions (modes) and approximate eigenfunctions (quasi-modes) of the Laplacian on a compact manifold $(M, g)$ with completely integrable geodesic flow. We give a new proof of the main result of [TZ that $(M, g)$ with integrable Laplacians and with uniformly bounded eigenfunctions must be flat. The proof is based on the use of Birkhoff normal forms and on a comparison of modes and quasimodes. In the process, we discuss tunnelling between resonant tori and give a proof that eigenfunctions concentrate on individual tori in the non-resonant case. We also give brief expositions of results in [TZ2, SZ].
\end{abstract}

\section{INTRODUCTION}

In this article we are interested in the semiclassical $h \rightarrow 0$ asymptotics of modes (eigenfunctions) $\phi_{j}(h)$

$$
\left(h^{2} \Delta+V\right) \phi_{j}=\lambda_{j}^{2}(h) \phi_{j}, \quad\left\langle\phi_{j}, \phi_{k}\right\rangle=\delta_{j k}
$$

of Schroedinger operators on a Riemannian manifold $(M, g)$. Usually we take $(M, g)$ to be compact for convenience, but equally interesting problems occur on non-compact manifolds such as $\mathbb{R}^{n}$. Many questions about eigenfunctions are motivated by modern mesoscopic physics and quantum chaos, in particular by the physics of quantum dots, wires and corrals (for a recent sample, see e.g. [FE, FHHMLE, [K, Mi, Mi2]). Let us mention just a few topics and recent articles from the mathematics and physics literature :

- Norms of eigenfunctions: $\left\|\phi_{j}\right\|_{L^{p}}$ [ABST, AS, Bour, D, [IS, [SZ;

- Eigenfunction scarring [AA, Be2, [B, RS, М];

- Volume and distribution of nodal surfaces $\left\{\phi_{j}=0\right\}$ [BGS, BS, CHENG, DF, DF2, JU;

- Number and distribution of critical points $\left\{\nabla \phi_{j}=0\right\}$ [JN, SZ];

- Matrix elements $\left\langle A \phi_{j}, \phi_{k}\right\rangle$ of pseudodifferential operators [KH, Z;

- Resonance and tunnelling between wells or tori [HS, HSu, M, Wi].

Most of the classical and more recent problems about eigenfunctions are believed to be very difficult. The main reason for the difficulty is that in general there are no explicit formulae for eigenfunctions or even for approximations to them. Lacking such explicit formulae, one has to rely on local approximations that lose track of the global geometry of $(M, g)$ or on wave equation or resolvent methods, which involve averages over many eigenfunctions. It is thus difficult to obtain global information about individual modes.

Date: November 19, 2001.

Research partially supported by NSERC grant \#OGP0170280

Research partially supported by NSF grant \#DMS-0100474. 
In this article, we would like to review and revisit some recent results about $L^{p}$-norms of eigenfunctions, obtained in part by the authors and in part by one of the authors in collaboration with C. Sogge. For some $(M, g), L^{p}$ - norms of eigenfunctions are extremally large [SZ and for some they are extremally small [TZ, TZ2]. The problems studied in [TZ, TZ2, SZ were to characterise metrics and potentials producing extremal growth rates of eigenfunctions. Of course, one would like to have some control over growth rates of eigenfunctions for any $(M, g, V)$. Our main focus in this article is to give a different and in some ways more natural proof of the results on $L^{\infty}$ norms of modes in the quantum integrable case of [TZ], based on the construction of quasimodes.

On a more expository level, we would also like to advertise quantum integrable systems as a simple setting for harmonic analysis. Quantum integrable Laplacians are generalizations of Laplacians on flat tori and of harmonic oscillators, and have most of the features which make these settings attractive for harmonic analysis. Yet they also bring in interesting new geometric and analytic phenomena. Most of the special eigenfunctions studied in the literature are quantum integrable, see e.g. "B.K.S, Bour, CV1, D, JN, KMS. To make the ideas more accessible to harmonic analysts, we describe a number of two-dimensional examples in some detail in Section (5).

To motivate the problem and result, let us consider the setting where we first learn about eigenfunctions, namely the flat torus $\mathbb{R}^{n} / L$ where $L$ is lattice such as $\mathbb{Z}^{n}$. The eigenfunctions $\phi_{\lambda}$ are of course the exponentials $e^{i\langle\lambda, x\rangle}$ where $\lambda \in L^{*}$, the dual lattice. The harmless looking facts

$$
\phi_{\lambda}(x)=e^{i\langle\lambda, x\rangle}, \quad\left|\phi_{\lambda}(x)\right|=1, \quad(\forall x)
$$

about the most familiar eigenfunctions raise the questions: when can eigenfunctions be expressed in this kind of WKB form $a(x) e^{i S(x) / h}$ or as an oscillatory integral? And how common is it that eigenfunctions have uniformly bounded sup norms?

Heuristic answers to these and other questions about eigenfunctions are suggested by correspondence principles between excited states of quantum systems and their underlying classical dynamics. The quantum Hamiltonian is simply the Laplacian $\Delta$, while its classical counterpart is its bicharacteristic flow, namely the geodesic flow $G^{t}: S^{*} M \rightarrow S^{*} M$. The clearest statements concern the most extreme dynamical regime, integrable systems and chaotic systems, with mixed and somewhat fuzzy statements about intermediate systems. The following description summarizes the current conjectural picture of eigenfunctions; it is not intended to be exhaustive. Eigenfunctions are described according to the regime of the corresonding classical dynamics.

- The Integrable regime: Approximate eigenfunctions may be constructed by a WKB method associated to invariant tori satisfying Bohr-Sommerfeld quantization conditions. Sequences of eigenfunctions localize in phase space on (unions of ) the invariant tori for the geodesic flow. They 'scar' (concentrate singularly) on the projections of those tori.

- The intermediate KAM regime: WKB quasimodes may be constructed for a positive measure set of "Cantori". There are regular and irregular modes corresponding to the Cantori and the regions of instability between them. 
- The chaotic regime: eigenfunctions behave like random waves, e.g. like random spherical harmonics. They do not localize in phase space, but rather become uniformly distributed, as do their critical points and zero sets. Only low-lying modes should scar.

Quasi-modes are essentially parametrices for modes, in some ways analogous to parametrices for operator kernels (e.g. the Hadamard parametrix for the heat or wave kernel). Unlike operator parametrices, however, they do not necessarily approximate individual modes. We return to this point below.

Quasi-modes are usually thought of as oscillatory integrals associated to geodesic-flow invariant Lagrangean (or isotropic) submanifolds of an energy level $\left\{H(x, \xi)=|\xi|_{g}^{2}+V(x)=\right.$ $E\} \subset T^{*} M$. There are axioms for quasimodes [CV2] which allow for more general constructions, in particular in the KAM case $[\mathrm{CV} 2, \mathbb{\mathrm { Q }}, \mathbb{P}]$. Existence of quasimodes associated to a Lagrangean submanifold $\Lambda$ involves a Bohr-Sommerfeld quantization condition on $\Lambda$ and some stability properties. For instance, there are always quasi-modes associated to stable closed geodesics [CV2, R, but there are no known quasi-mode constructions associated to unstable closed geodesics. For this reason, quasi-mode constructions are most effective in integrable or KAM systems, and these regimes represent the best chance of understanding individual eigenfunctions. In the opposite chaotic regime, there are no known quasi-modes and heuristic reasons suggest there aren't any explicitly constructible approximate solutions. Indeed, the modes are believed to behave like random waves and to be rather featureless. Finally, in the no man's land between the nearly integrable and the quite chaotic, there are few if any heuristics or results of a global nature. The only known results are obtained by piecing together local information on eigenfunctions.

Let us now state some recent rigorous results. First we need some notation: by $\Psi^{m}(M)$ we denote the space of mth order pseudodifferential operators over $M$. We say that an operator $P \in \Psi^{1}(M)$ is quantum integrable if there exist $P_{2}, \ldots, P_{m} \in \Psi^{1}(M)$ such that $\left[P_{j}, P_{k}\right]=0$ and such that $P_{1}^{2}+\cdots+P_{m}^{2}$ is elliptic. We denote by $p_{k}=\sigma_{P_{k}}$ the principal symbol of $P_{k}$. Since $\sigma_{\left[P_{i}, P_{j}\right]}=\left\{p_{i}, p_{j}\right\}$ (the Poisson bracket), it follows that the $p_{j}$ 's generate a homogeneous Hamiltonian action $\Phi_{t}$ of $t \in \mathbb{R}^{n}$ on $T^{*} M-0$ with moment map

$$
\mathcal{P}: T^{*} M-0 \rightarrow \mathbb{R}^{n}, \quad \mathcal{P}=\left(p_{1}, \ldots, p_{n}\right) .
$$

We denote the image $\mathcal{P}\left(T^{*} M-0\right)$ by $B$, by $B_{\text {reg }}$ (resp. $B_{\text {sing }}$ ) the regular values (resp. singular values) of the moment map.

We are going to impose a 'finite complexity' assumption on the classical integrable system. Suffice it to say that it holds for all systems of interest in physics: for each $b=\left(b^{(1)}, \ldots, b^{(n)}\right) \in$ $B$, let $m_{c l}(b)$ denote the number of $\mathbb{R}^{n}$-orbits of the joint flow $\Phi_{t}$ on the level set $\mathcal{P}^{-1}(b)$. Then

$$
\text { Finite complexity condition : } \exists M: m_{c l}(b)<M \quad(\forall b \in B) \text {. }
$$

When $b \in B_{\text {reg }}$, then $\mathcal{P}^{-1}(b)$ is the union of $m_{c l}(b)$ isolated Lagrangian tori. If $b \in B_{\text {sing }}$, then $\mathcal{P}^{-1}(b)$ consists of a finite number of connected components, each of which is a finite union of orbits. These orbits may be Lagrangian tori, singular compact tori (ie. compact tori of dimension $<n$ ), or non-compact orbits consisting of cylinders or planes. 
On the quantum level we will assume:

$$
\text { Bounded eigenvalue multiplicity : } \exists M^{\prime}: m(\lambda) \leq M^{\prime} \quad\left(\forall \lambda ; m(\lambda)=\operatorname{dim} V_{\lambda}\right) .
$$

This reduces estimates of eigenfunctions to estimates of joint eigenfunctions of the commuting operators. When unbounded multiplicities occur, such as on $\mathbb{R}^{n} / \mathbb{Z}^{n}$, then it is well-known that one may construct sequences of eigenfunctions with unbounded sup norms even on flat tori.

THEOREM 0.1. Suppose that $\Delta$ is a quantum completely integrable Laplacian on a compact

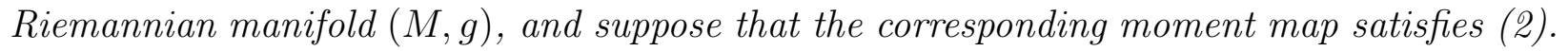
Then: if the $\Delta$-eigenfunctions have uniformly bounded $L^{\infty}$ norms, then $(M, g)$ is flat.

More generally, suppose that $\hbar^{2} \Delta+V$ is a quantum completely integrable Schroedinger operator, and that the corresponding moment map $\mathcal{P}$ is proper and satisfies (2). Assume there exists an energy level $E$ such that the eigenfunctions have uniformly bounded $L^{\infty}$ norms as $h \rightarrow 0$. Then: if $E>\max V$, and $(M,(E-V) g)$ is flat. If (a) (or (b)) holds for all energy levels $E$ in an interval $E_{1}<E<E_{2}$, then $(M, g)$ is flat and $V$ is constant.

To prove the result, we must relate the analysis of modes to the geometry of the Liouville foliation (by invariant tori) of $T^{*} M$ of the completely integrable system. In [TZ], this relation was brought out through trace formulae. In this article, we approach it through quasimodes. With some work, we believe the results should generalize to some degree to quantum KAM systems, i.e. to small perturbations of completely integrable systems. However, we leave that extension for the future.

Roughly speaking, the idea is this: Quasi-modes are directly associated to the individual Lagrangian tori of the completely integrable system. Hence we can directly relate norms of quasimodes to geometry of the tori. To draw conclusions about norms of modes, we must then relate modes and quasimodes. Since the classic article "Modes and Quasi-modes" A] of V. I. Arnol'd (also based on examples due to A. I. Schnirelman), attention has been drawn to the danger of of drawing conclusions about modes from conclusions about quasimodes. As Arnold cautioned, modes are not always approximated by quasimodes, but rather may be linear combinations of quasimodes with close quasi-eigenvalues. If the linear combinations involve 'many' closely spaced quasi-eigenvalues, then the connection between modes and quasi-modes becomes blurred. However, this danger mainly pertains to scalar spectra, i.e. the spectrum of one operator; it does not occur in the approximation of joint eigenfunctions of a completely integrable set of operators by joint quasimodes, since the joint spectrum in $\mathbb{R}^{n}$ is much better separated than the scalar spectrum of the Hamiltonian alone. For the joint spectrum, a simple non-resonance condition is sufficient to ensure that modes are even well-approximated by individual quasi-modes.

When modes of integrable systems can be connected with invariant tori for the geodesic flow, one can hope to relate growth rates of $L^{p}$-norms of sequences of eigenfunctions to singularities of projections of the associated tori. For instance, as will be discussed further in Section (5), invariant eigenfunctions on surfaces of revolution correspond to to invariant 
torus in $S^{*} S^{2}$ consisting of meridian geodesics between the poles. This torus has a "blowdown" singularity under the natural projection $\pi: S^{*} S^{2} \rightarrow S^{2}$, i.e. the fiber $S_{N P}^{*} S^{2} \rightarrow\{N P\}$ over the north pole is blown-down to a point under the projection. This kind of singularity causes the largest concentration of the mass of a mode at a point; hence the invariant eigenfunctions which correspond to the meridian torus are (at least in order of magnitude) extremals for the $L^{\infty}$ and other $L^{p}$ norms for sufficiently large $p$. Other important tori in the $L^{p}$ theory are the singular tori where the torus drops dimension, e.g. the equators of a surface of revolution. Eigenfunctions concentrated on such singular tori are (in order of magnitude) extremals for low $L^{p}$ norms: although they do not blow up as much, they blow up on a set of larger measure and hence are favorable for $L^{p}$ norm blow-up.

Let us now recall some other recent results; we refer to [TZ2, SZ for further discussion.

The next result also pertains to quantum completely integrable systems. We impose a stronger hypothesis on the classical system, namely Eliasson non-degeneracy (see [TZ2 for background). We then obtain quantitative results on the $L^{p}$ blow-up of eigenfunctions in non-flat cases, i.e. in cases where (by the previous theorem) some blow-up must occur. We emphasize that our notation for eigenvalues (1) is such that $\Delta \phi_{k}=\lambda_{k}^{2} \phi_{k}$.

THEOREM 0.2. Suppose that $(M, g)$ is a compact Riemannian manifold with completely integrable geodesic flow satisfying Eliasson's non-degeneracy condition. Then $(M, g)$ is non-flat if and only if for every $\epsilon>0$, there exists a sequence of $L^{2}$-normalized eigenfunctions $\phi_{k}$ satisfying:

$$
\left\{\begin{array}{l}
\left\|\phi_{k}\right\|_{\infty} \geq C(\epsilon) \lambda_{k}^{\frac{1}{4}-\epsilon} . \\
\left\|\phi_{k}\right\|_{p} \geq C(\epsilon) \lambda_{k}^{\frac{p-2}{4 p}-\epsilon}
\end{array}\right.
$$

Modulo the $\epsilon$ 's, these estimates are sharp. They are based on the existence of codimension one singular leaves of the Lagrangian foliation, and on the construction of precise quasimodes attached to non-degenerate singular leaves. In general, a completely integrable system does not have higher codimension singular leaves (e.g. one may take the product $M=\mathbf{T}^{n-2} \times S^{2}$ of a flat torus and a surface of revolution to obtain completely integrable $(M, g)$ of any dimension whose only singular leaves are of codimension one). When higher codimension leaves occur, the blow-up rate increases. In the case where $(M, g)$ has a one-dimensional singular leaf, then our methods show that there exists a sequence of eigenfunctions satisfying: $\left\|\phi_{k}\right\|_{\infty} \geq C(\epsilon) \lambda_{k}^{\frac{n-1}{4}-\epsilon}$. Such a one-dimensional leaf is simply a stable elliptic geodesic $\gamma$ which is fixed by the other Hamiltonian flows of the completely integrable system. This estimate is comparable to the recent estimate of Donnelly $[\mathbb{D}$ on the blow-up rate of a sequence of eigenfunctions on certain $(M, g)$ with an isometric $S^{1}$ action. Note that Donnelly denotes the eigenvalue by $\lambda$ while we denote it by $\lambda^{2}$. Donnelly also assumes the existence of a non-degenerate singular orbit $\gamma$ of the joint action of the geodesic flow and $S^{1}$ action. Such a $\gamma$ is a a stable closed geodesic, such as the equator on a convex surface of revolution, which is invariant under the $S^{1}$ action. The corresponding sequence of eigenfunctions is actually a sequence of singular quasi-modes associated to $\gamma$. Thus, it is the same phase space phenomenon in either setting which is responsible for this common blow-up rate. 
We now turn to results by one of the authors with C. Sogge on the opposite extreme: which $(M, g)$ have maximal eigenfunction growth? It follows from classical remainder estimates of Avakumovic and Hormander that $L^{2}$-normalized eigenfunctions $\Delta \phi_{\lambda}=\lambda^{2} \phi_{\lambda}$ have $L^{\infty}$-norms satisfying

$$
\left\|\phi_{\lambda}\right\|_{\infty} \leq C \lambda^{\frac{n-1}{2}}, \quad n=\operatorname{dim} M
$$

When a sequence of eigenfunctions of $(M, g)$ actually achieves this bound, we say that $(M, g)$ has maximal eigenfunction growth. The main result of [SZ implies a necessary condition on a compact Riemannian manifolds $(M, g)$ with maximal eigenfunction growth: there must exist a point $x \in M$ for which the set

$$
\mathcal{L}_{x}=\left\{\xi \in S_{x}^{*} M: \exists T: \exp _{x} T \xi=x\right\}
$$

of directions of geodesic loops at $x$ has positive surface measure. Here, exp is the exponential map, and the measure $|\Omega|$ of a set $\Omega$ is the one induced by the metric $g_{x}$ on $T_{x}^{*} M$. For instance, the poles $x_{N}, x_{S}$ of a surface of revolution $\left(S^{2}, g\right)$ satisfy $\left|\mathcal{L}_{x}\right|=2 \pi$. More generally, we may consider maximal $L^{p}$ growth of eigenfunctions. The universal esimates in this case are due to Sogge So1, So2].

TheOREM 0.3. If $\left|\mathcal{L}_{x}\right|=0$ for every $x \in M$ then

$$
\frac{\|\phi\|_{L^{p}(M)}}{\|\phi\|_{L^{2}(M)}}=o\left(\lambda^{\delta(p)}\right), \quad p>\frac{2(n+1)}{n-1}
$$

where

$$
\delta(p)=\left\{\begin{array}{l}
n\left(\frac{1}{2}-\frac{1}{p}\right)-\frac{1}{2}, \quad \frac{2(n+1)}{n-1} \leq p \leq \infty \\
\frac{n-1}{2}\left(\frac{1}{2}-\frac{1}{p}\right), \quad 2 \leq p \leq \frac{2(n+1)}{n-1} .
\end{array}\right.
$$

It is proved that generic metrics $g$ on any $M$ satisfy the hypothesis of having zero-measure loop directions at every point. Hence, maximal eigenfunction growth is a rare condition. The strongest conclusion is available for real analytic Riemannian manifolds:

THEOREM 0.4. Suppose that $(M, g)$ is a real analytic with maximal eigenfunction growth. Then there exists a point $m \in M$ such that all geodesics issuing from the point $m$ return to $m$ at the same time $\ell$. In particular, if $\operatorname{dim} M=2$, then $M$ is topologically a 2-sphere $S^{2}$ or a real projective plane $\mathbb{R} P^{2}$.

It would be interesting to investigate maximal eigenfunction growth in small $L^{p}$ norms. It is explained in So1, So2 that the geometry of eigenfunctions changes at the critical

$p=\frac{2(n+1)}{n-1}$, and that sizes of loop directions is no longer relevant. In the case of the found sphere, at this value of $p$ the extremals for the $L^{p}$ norm (at least in order of magnitude) shift from eigenfunctions which localize at a point (zonal spherical harmonics) to eigenfunctions which localize on a closed geodesic (highest weight spherical harmonics). It is possible that maximal $L^{p}$ growth in the small range of $p$ is related to existence of the same stable elliptic geodesics $\gamma$ which gave rise to the $L^{p}$ estimates in [TZ2, D].

\section{QuANTUM InTEGRABLE SYSTEMS}

We begin by recalling the definition of a completely integrable system. 
1.1. Completely integrable systems. By a completely integrable system on $T^{*} M$ we mean a set of $n$ independent, $C^{\infty}$ functions $p_{1}, \ldots, p_{n}$, on $T^{*} M$ satisfying:

- $\left\{p_{i}, p_{j}\right\}=0$ for all $1 \leq i, j \leq n$;

- $d p_{1} \wedge d p_{2} \wedge \cdots \wedge d p_{n} \neq 0$ on an open dense subset of $T^{*} M$.

The associated moment map is defined by

$$
\mathcal{P}=\left(p_{1}, \ldots, p_{n}\right): T^{*} M \rightarrow B \subset \mathbb{R}^{n} .
$$

We refer to to the set $B$ as the 'image of the moment map.' The Hamiltonians generate an action of $\mathbb{R}^{n}$ defined by

$$
\Phi_{t}=\exp t_{1} \Xi_{p_{1}} \circ \exp t_{2} \Xi_{p_{2}} \cdots \circ \exp t_{n} \Xi_{p_{n}}
$$

We often denote $\Phi_{t}$-orbits by $\mathbb{R}^{n} \cdot(x, \xi)$. The isotropy group of $(x, \xi)$ will be denoted by $\mathcal{I}_{(x, \xi)}$. When $\mathbb{R}^{n} \cdot(x, \xi)$ is a compact Lagrangean orbit, then $\mathcal{I}_{(x, \xi)}$ is a lattice of full rank in $\mathbb{R}^{n}$, and is known as the 'period lattice', since it consists of the 'times' $T \in \mathbb{R}^{n}$ such that $\left.\Phi_{T}\right|_{\Lambda^{(j)}(b)}=I d$.

We will need the following:

DeFinition 1.1. We say that:

- $b \in B_{\text {sing }}$ if $\mathcal{P}^{-1}(b)$ is a singular level of the moment map, i.e. if there exists a point $(x, \xi) \in \mathcal{P}^{-1}(b)$ with $d p_{1} \wedge \cdots \wedge d p_{n}(x, \xi)=0$. Such a point $(x, \xi)$ is called a singular point of $\mathcal{P}$.

- a connected component of $\mathcal{P}^{-1}(b)\left(b \in B_{\text {sing }}\right)$ is a singular component if it contains a singular point;

- an orbit $\mathbb{R}^{n} \cdot(x, \xi)$ of $\Phi_{t}$ is singular if it is non-Lagrangean, i.e. has dimension $<n$;

- $b \in B_{\text {reg }}$ and that $\mathcal{P}^{-1}(b)$ is a regular level if all points $(x, \xi) \in \mathcal{P}^{-1}(b)$ are regular, i.e. if $d p_{1} \wedge \cdots \wedge d p_{n}(x, \xi) \neq 0$.

- a component of $\mathcal{P}^{-1}(b)\left(b \in B_{\text {sing }} \cup B_{\text {reg }}\right)$ is regular if it contains no singular points.

By the Liouville-Arnold theorem $[\mathrm{AM}]$, the orbits of the joint flow $\Phi_{t}$ are diffeomorphic to $\mathbb{R}^{k} \times T^{m}$ for some $(k, m), k+m \leq n$. By the properness assumption on $\mathcal{P}$, a regular level has the form

$$
\mathcal{P}^{-1}(b)=\Lambda^{(1)}(b) \cup \cdots \cup \Lambda^{\left(m_{c l}\right)}(b), \quad\left(b \in B_{r e g}\right)
$$

where each $\Lambda^{(l)}(b) \simeq T^{n}$ is an $n$-dimensional Lagrangian torus. The classical (or geometric) multiplicity function $m_{c l}(b)=\# \mathcal{P}^{-1}(b)$, i.e. the number of orbits on the level set $\mathcal{P}^{-1}(b)$, is constant on connected components of $B_{\text {reg }}$ and the moment map (7) is a fibration over each component with fiber (8). In sufficiently small neighbourhoods $\Omega^{(l)}(b)$ of each component torus, $\Lambda^{(l)}(b)$, the Liouville-Arnold theorem also gives the existence of local action-angle variables $\left(I_{1}^{(l)}, \ldots, I_{n}^{(l)}, \theta_{1}^{(l)}, \ldots, \theta_{n}^{(l)}\right)$ in terms of which the joint flow of $\Xi_{p_{1}}, \ldots, \Xi_{p_{n}}$ is linearized $[\mathrm{AM}]$. For convenience, we henceforth normalize the action variables $I_{1}^{(l)}, \ldots, I_{n}^{(l)}$ so that $I_{j}^{(l)}=$ $0 ; j=1, \ldots, n$ on the torus $\Lambda^{(l)}(b)$.

Quantum integrability involves semiclassical pseudo-differential operators, so let us pause to set up the notation. Given an open $U \subset \mathbb{R}^{n}$, we say that $a(x, \xi ; \hbar) \in C^{\infty}\left(U \times \mathbb{R}^{n}\right)$ is in 
the symbol class $S^{m, k}\left(U \times \mathbb{R}^{n}\right)$, provided

$$
\left|\partial_{x}^{\alpha} \partial_{\xi}^{\beta} a(x, \xi ; \hbar)\right| \leq C_{\alpha \beta} \hbar^{-m}(1+|\xi|)^{k-|\beta|} .
$$

We say that $a \in S_{c l}^{m, k}\left(U \times \mathbb{R}^{n}\right)$ provided there exists an asymptotic expansion:

$$
a(x, \xi ; \hbar) \sim \hbar^{-m} \sum_{j=0}^{\infty} a_{j}(x, \xi) \hbar^{j},
$$

valid for $|\xi| \geq \frac{1}{C}>0$ with $a_{j}(x, \xi) \in S^{0, k-j}\left(U \times \mathbb{R}^{n}\right)$ on this set. We denote the associated $\hbar$ Kohn-Nirenberg quantization by $O p_{\hbar}(a)$, where this operator has Schwartz kernel given locally by the formula:

$$
O p_{\hbar}(a)(x, y)=(2 \pi \hbar)^{-n} \int_{\mathbb{R}^{n}} e^{i(x-y) \xi / \hbar} a(x, \xi ; \hbar) d \xi .
$$

By using a partition of unity, one constructs a corresponding class, $O p_{\hbar}\left(S^{m, k}\right)$, of properlysupported $\hbar$-pseudodifferential operators acting on $C^{\infty}(M)$.

Definition 1.2. We say that the operators $Q_{j} \in O p_{\hbar}\left(S_{c l}^{m, k}\right) ; j=1, \ldots, n$, generate a semiclassical quantum completely integrable system if

$$
\left[Q_{i}, Q_{j}\right]=0 ; \forall 1 \leq i, j \leq n,
$$

and the respective semiclassical principal symbols $q_{1}, \ldots, q_{n}$ generate a classical integrable system with $d q_{1} \wedge d q_{2} \wedge \cdots \wedge d q_{n} \neq 0$ on a dense open set $\Omega \subset T^{*} M-0$. We also assume that the finiteness condition ( $(\mathcal{G})$ is satisfied.

Let us pause to relate this notion to the possibly more familiar notion of $n$ commuting (ordinary) pseudo-differential operators $P_{1}, \ldots, P_{n}$. Such operators have a joint spectrum. In eigenvalue asymptotics for a joint spectrum, it is natural to consider ladders of eigenvalues:

$$
\left\{\left(\lambda_{1 k}, \ldots, \lambda_{n k}\right) \in \operatorname{Spec}\left(P_{1}, \ldots, P_{n}\right) ; \forall j=1, . ., n, \lim _{k \rightarrow \infty} \frac{\lambda_{j k}}{\left|\lambda_{k}\right|}=b_{j}\right\}
$$

where $\left|\lambda_{k}\right|:=\sqrt{\lambda_{1 k}^{2}+\ldots+\lambda_{n k}^{2}}$. We then introduce the semiclassical parameter $\hbar$ with values in a sequence $\left\{\hbar_{k}^{-1} ; k=1,2,3, \ldots\right\}$ with $\hbar_{k} \rightarrow 0$ as $k \rightarrow \infty$. Now, consider the semiclassically scaled operators:

$$
Q_{j}:=\hbar P_{j} ; \quad j=1,2, \ldots, n .
$$

In the semiclassical regime, it is convenient to work with the $Q_{j}$ 's rather than the $P_{j}$ 's. For the eigenfunctions $\phi_{b, \hbar}$ associated with the ladder (9) we clearly have:

$$
Q_{j} \phi_{b, \hbar}=b_{j} \phi_{b, \hbar}+\mu_{j k} \phi_{b, \hbar}, \text { where } \mu_{j k}=o(1) \text { as } \hbar \rightarrow 0 \text {. }
$$

The operators $Q_{j} ; j=1, \ldots, n$ are semiclassical pseudodifferential operators.

Consequently, in the homogeneous case, the operators $P_{1}=\sqrt{\Delta}, P_{2}, \ldots, P_{n}$ are classical pseudodifferential operators of order one, it readily follows that $Q_{j}:=\hbar P_{j} \in O p\left(S_{c l}^{0,1}\right)$ generate a semiclassical quantum integrable system in the sense of Definition 1.2 . 


\section{QuASIMOdes AND BIRKhOFF NORMAL FORMS}

First, some notation. Following the convention in [CP], we denote $\hbar$-microlocal equivalence on an open set, $\Omega \subset T^{*} M$ by $=_{\Omega}$. Also, recall that [CP] a family of distributions, $u_{\hbar}$, where $\hbar \in\left(0, \hbar_{0}\right]$ is called admissible if for any cutoff function, $\chi(x, \xi) \in C_{0}^{\infty}(\Omega)$, there is an $N \in \mathbb{Z}$ and $s \in \mathbb{Z}$, such that

uniformly for $\hbar \in\left(0, \hbar_{0}\right]$.

$$
\left\|\hbar^{N} O p_{\hbar}(\chi) u_{\hbar}\right\|_{H^{-s}}=\mathcal{O}(1)
$$

2.1. Inverse Birkhoff normal form. All of our results on localization of modes and quasimodes are based on the use of Birkhoff normal forms. We begin by defining such normal forms.

Let $b \in B_{\text {reg }}$ and consider the regular level set $\mathcal{P}^{-1}(b)$ of the moment map with $\mathcal{L}^{(l)}$ denote the pull-back of the Maslov line bundle over the affine torus, $\mathbb{T}^{n}$, given by $I_{1}^{(l)}=\ldots=I_{n}^{(l)}=0$ and parametrized by the angle variables $\theta_{1}^{(l)}, \ldots, \theta_{n}^{(l)}$. We denote the space of smooth sections of this bundle by $C^{\infty}\left(\mathbb{T}^{n} ; \mathcal{L}^{(l)}\right)$. The image of $\Omega^{(l)}(b)$ under the map to normal form will be denoted by $\Omega_{0}^{(l)}=\mathbb{T}^{n} \times D_{1}$, where $D_{1}$ denotes a sufficiently small neighbourhood of $0 \in \mathbb{R}^{n}$. We start with a quantum normal-form construction which can be found in a somewhat different form in CV2].

LEMma 2.1. For $l=1, \ldots, k$ and $j=1, \ldots, n$, there exist $\hbar$-Fourier integral operators, $U_{b}^{(l)}$ : $C^{\infty}(M) \rightarrow C^{\infty}\left(\mathbb{T}^{n} ; \mathcal{L}^{(l)}\right)$, microlocally elliptic on $\Omega^{(l)}$, together with $C^{\infty}$ symbols, $f_{j}^{(l)}(x ; \hbar) \sim$ $\sum_{k=0}^{\infty} f_{j k}^{(l)}(x) \hbar^{k}$, with $f_{j 0}(0)=0$ such that:

$$
U^{(l) *} f_{j}^{(l)}\left(Q_{1}-b_{1}, \ldots, Q_{n}-b_{n} ; \hbar\right) U^{(l)}={ }_{\Omega_{0}^{(l)}} \frac{\hbar}{i} \frac{\partial}{\partial \theta_{j}} .
$$

Moreover, when $Q_{1}, \ldots, Q_{n}$ are formally self-adjoint, the operator $U_{b}^{(l)}$ can be taken to be microlocally unitary.

Proof: To simplify the writing a little, we drop the superscript $(l)$ in the following and denote the $\hbar$ principal symbols of $Q_{1}, \ldots, Q_{n}$ by $q_{1}, \ldots, q_{n}$. Let $\kappa: \Omega(b) \rightarrow \mathbb{T}^{n} \times D_{1}$ be a canonical map with the property that,

$$
q_{j} \circ \kappa(\theta, I)=\tilde{q}_{j}(I), \quad j=1, \ldots, n,
$$

for some $\tilde{q}_{j} \in C^{\infty}\left(D_{1}\right)$. Then, since $d q_{1}, \ldots, d q_{n}$ are linearly-independent on $\Omega(b)$, by the inverse function theorem, there exists $f_{j 0} \in C^{\infty}\left(\mathbb{R}^{n}\right)$ with $f_{j 0}(0)=0$ such that:

$$
f_{j 0}\left(q_{1}-b_{1}, \ldots, q_{n}-b_{n}\right) \circ \kappa=I_{j}, \quad j=1, \ldots, n .
$$

By the semiclassical Egorov Theorem, there exists an $\hbar$-Fourier integral operator $U_{0}$ : $L^{2}\left(\mathbb{T}^{n} ; \mathcal{L}\right) \rightarrow L^{2}(M)$ quantizing $\kappa$ such that for $j=1, \ldots, n$,

$$
U_{0}^{-1} f_{j 0}\left(Q_{1}-b_{1}, \ldots, Q_{n}-b_{n}\right) U_{0}={ }_{\mathbb{T}^{n} \times D_{1}} O p_{\hbar}\left(I_{j}\right)+\hbar O p_{\hbar}\left(r_{1}(\theta, I)\right) .
$$

Recall that $L^{2}\left(\mathbb{T}^{n} ; \mathcal{L}\right)$ has a natural Hilbert basis given by the shifted exponentials, $e_{m}(x)=$ $\exp (i\langle m+\nu / 4, x\rangle)$, with $m \in \mathbb{Z}^{n}$. The next step involves reduction of the order of the error term on the RHS of (13). We do this by making Fourier series expansions of all symbols in 
terms of the shifted exponentials, $e_{m}(x)$, and conjugating the expression on the RHS of (13) by $U_{1}:=\exp \left(i \hbar O p_{h}\left(v_{1}\right)\right)$ where $v_{1}(\theta, I) \in C^{\infty}\left(\mathbb{T}^{n} \times D_{1}\right)$ :

$$
\begin{array}{r}
\exp \left[-i \hbar O p_{\hbar}\left(v_{1}\right)\right] \circ\left[O p_{\hbar}\left(I_{j}\right)+\hbar O p_{\hbar}\left(r_{1}\right)\right] \circ \exp \left[i \hbar O p_{\hbar}\left(v_{1}\right)\right] \\
={ }_{\Omega}\left[I d-i \hbar O p_{\hbar}\left(v_{1}\right)\right] \circ\left[O p_{\hbar}\left(I_{j}\right)+\hbar O p_{\hbar}\left(r_{1}\right)\right] \circ\left[I d+i \hbar O p_{\hbar}\left(v_{1}\right)\right]+\mathcal{O}\left(\hbar^{2}\right) .
\end{array}
$$

Note that, by the pseudodifferential symbolic calculus,

$\left[I d-i \hbar O p_{\hbar}\left(v_{1}\right)\right] \circ\left[O p_{\hbar}\left(I_{j}\right)+\hbar O p_{\hbar}\left(r_{1}\right)\right] \circ\left[I d+i \hbar O p_{\hbar}\left(v_{1}\right)\right]+\mathcal{O}\left(\hbar^{2}\right)=\mathbb{T}^{n} \times D_{1} O p_{\hbar}\left(I_{j}\right)+\hbar O p_{\hbar}\left(e_{1 j}\right)+\mathcal{O}\left(\hbar^{2}\right)$

Clearly, to get rid of the $O p_{\hbar}\left(e_{1 j}\right)$ term on the RHS of (15), we would like to solve the following transport equations for $v_{1}$ :

$$
e_{1 j}(\theta, I)=\left\{v_{1}, I_{j}\right\}(\theta, I)+r_{1}(\theta, I) .
$$

Keeping in mind that all operators are acting on $L^{2}\left(\mathbb{T}^{n} ; \mathcal{L}\right)$, it follows that these equations can be rewritten in the form:

$$
\frac{\partial}{\partial \theta_{j}} v_{1}(\theta, I)-\frac{\nu}{4} v_{1}(\theta, I)+r_{1}(\theta, I)=0 \quad ; j=1, \ldots, n .
$$

However, in order to solve (17), we have to subtract off the zeroth shifted Fourier coefficient, $\tilde{f}_{j 1}(I)$, of $r_{1}(\theta, I)$, and then match Fourier coefficients on both sides of (17). Consequently, if

$$
f_{j 1}\left(q_{1}-b_{1}, \ldots, q_{n}-b_{n}\right) \circ \kappa=\tilde{f}_{j 1}\left(I_{1}, \ldots, I_{n}\right),
$$

it follows from (13)-(17) above that for $j=1, \ldots, n$,

$U_{1}^{-1} U_{0}^{-1} \circ\left[f_{0 j}\left(Q_{1}-b_{1}, \ldots, Q_{n}-b_{n}\right)+\hbar f_{1 j}\left(Q_{1}-b_{1}, \ldots, Q_{n}-b_{n}\right)\right] \circ U_{0} U_{1}=_{\mathbb{T} \times D_{1}} O p_{\hbar}\left(I_{j}\right)+\mathcal{O}\left(\hbar^{2}\right)$.

To finish the proof of the lemma, simply apply induction where the inductive step is exactly the same as the argument for the first step given above.

2.2. Quasimodes. In this section, we introduce the quasimodes $\psi_{b, \hbar}^{(l)}(x):=U_{b}^{(l)} u_{\hbar}(x)$.

Suppose that $\phi_{b, \hbar}$ is a joint eigenfunction of $Q_{1}, \ldots, Q_{n}$ with $Q_{j} \phi_{b, \hbar}=\left(b_{j}+\mu_{j}(\hbar)\right) \phi_{b, \hbar}$, where $\mu_{j}(\hbar)=o(1)$. Then, as a consequence of the normal form in Lemma $(2.1), u_{\hbar}=\Omega_{\Omega^{(l)}} U^{(l) *} \phi_{b, \hbar}$ is an admissible, microlocal solution of the system of equations:

$$
\frac{\hbar}{i} \frac{\partial}{\partial \theta_{j}} u_{\hbar}={ }_{\Omega_{0}^{(l)}} m_{j}(\hbar) u_{\hbar}, \quad j=1,2, \ldots, n .
$$

where, $m_{j}(\hbar) \in \hbar\left(\mathbb{Z}+\frac{\pi}{2}\left[\nu^{(l)}\right]\right)+\mathcal{O}\left(\hbar^{\infty}\right)$ and $m_{j}(\hbar)=o(1)$ as $\hbar \rightarrow 0$.

Proposition 2.2. The space of admissible microlocal solutions of the system of equations in (19) is one-dimensional. Consequently, the functions

$$
\left.u_{\hbar}(\theta)=\exp \left[i m_{1}(\hbar) \theta_{1}+\ldots+i m_{n}(\hbar) \theta_{n}\right)\right]
$$

span over $\mathbb{C}(\hbar)$ the space of admissible, microlocal solutions to (19). 
Proof: Let $u_{\hbar}$ be an admissible, microlocal solution to (19), by using Fourier transforms and cutting-off in the frequency variables [CP], one readily shows that there exists a global distributional solution, $v_{\hbar} \in \mathcal{D}^{\prime}\left(\mathbb{T}^{n} ; \mathcal{L}\right)$, to $(\mathbb{1 9})$ with $v_{\hbar}=\Omega_{\Omega_{0}} u_{\hbar}$. But then, by a simple quadrature, we have that $\left.v_{\hbar}=c(\hbar) \exp \left[i\left(m_{1}(\hbar)\right) \theta_{1}+\ldots++m_{n}(\hbar) \theta_{n}\right)\right]+\mathcal{O}\left(\hbar^{\infty}\right)$ for some $c(\hbar) \in \mathbb{C}(\hbar)$.

Proposition (2.2) has a natural corollary that characterizes the space of the so-called semiclassical quasimodes [CV2] attached to a component torus, $\Omega^{(l)}(b)$ :

COROLlary 2.3. The space of admissible, microlocal solutions to the system of equations

$$
Q_{j} \phi_{b, \hbar}={ }_{\Omega^{(l)}}\left(b_{j}+\mu_{j k}(\hbar)\right) \phi_{b, \hbar}+\mathcal{O}\left(\hbar^{\infty}\right) ; j=1, \ldots, n
$$

is spanned over $\mathbb{C}(\hbar)$ by the functions

$$
\psi_{b, \hbar}^{(l)}(x):=U_{b}^{(l)} u_{\hbar}(x),
$$

where, $U_{b}^{(l)}$, is the $\hbar$-Fourier integral operator constructed in Lemma (2.1) which depends locally regularly on $b \in B_{\text {reg }}$ and $u_{\hbar}(\theta)=\exp \left[i\left(m_{1}(\hbar) \theta_{1}+\ldots+m_{n}(\hbar) \theta_{n}\right)\right]$, with $m_{j}(\hbar) \in$ $\mathbb{Z}+\frac{\pi}{2}\left[\nu^{(l)}\right]+\mathcal{O}\left(\hbar^{\infty}\right)$.

Proof: The corollary follows immediately from Proposition (2.2) together with the Birkhoff normal form construction in Lemma (2.1).

Remark: By applying the lemma of stationary phase in the explicit expressions for the functions $U_{b}^{(l)} u_{\hbar}(x)$ on a sufficiently small open set $V \in \mathbb{R}^{n}$, it follows that there exist locallydefined phase functions, $\phi_{l}(x, \xi) \in C^{\infty}\left(V \times \mathbb{R}^{N}\right)$, parametrizing the Lagrangian torus, $\Lambda^{(l)}(b)$, and classical symbols $b_{l}(x, \xi ; \hbar) \sim \sum_{j} b_{j l}(x, \xi) \hbar^{j}$, such that:

$$
\psi_{b, \hbar}^{(l)}(x)=(2 \pi \hbar)^{-N / 2} \int_{\mathbb{R}^{N}} e^{i \phi_{l}(x, \xi) / \hbar} b_{l}(x, \xi ; \hbar) d \xi .
$$

These oscillatory integrals are precisely the usual semiclassical Lagrangian distributions (or "quasimodes") attached to Lagrangian tori.

We still have to determine the asymptotic formulas for the eigenvalues, $b_{j}+\mu_{j}(\hbar)$. These formulas are usually referred to as the (regular) Bohr-Sommerfeld quantization rules associated with a component Lagrangian torus:

\section{RESONANT TORI AND LOCALIZATION OF MODES}

The main results (Lemmas (3.1) and (3.2) of this section concern the localization of modes on tori. When the fiber

$$
\mathcal{P}^{-1}(b)=\Lambda^{(1)}(b) \cup \cdots \cup \Lambda^{\left(m_{c l}\right)}(b),
$$

where the $\Lambda^{(l)}(b) ; l=1, \ldots, m$ are has multiple components, it is only true in general that the joint eigenfunctions $\phi_{\lambda}$ with approximately the joint eigenvalue $\lambda$ concentrate on the union of the tori. The tunnelling problem which concerns us is the following:

Problem How is the mass of $\phi_{\lambda}$ distributed among the components $\Lambda^{(1)}(b)$ of $\mathcal{P}^{-1}(b)$ ?

In the case of a symmetric double well potential $V(x)=V(-x)$ on $\mathbb{R}$, it is well-known that eigenfunctions are either even or odd, and hence have their mass equally distributed in 
both wells. A similar phenomenon occurs in any integrable system with symmetries. In this case, the wells are resonant, i.e. the germs of the Hamiltonian flow at the two tori (or wells) are symplectically equivalent.

Resonance causes trouble when comparing sup norms of modes and quasi-modes, and hence when relating sup norms of modes to the geometry of invariant tori. However, this is an exceptional situation: we prove that in generic (non-resonant) situations, modes are well-approximated by individual quasimodes and localize on individual tori. To deal with the remaining resonant cases, we prove a result (Lemma (4.1)) which allows us to transfer attention from modes to quasimodes in the proof of Theorem (0.1). Namely, we prove that uniform boundedness of sup norms of modes implies the same for quasimodes ( Lemma (4.1)).

Much of this material is implicitly known (it appears in a slightly different form in [CV2, CP] in the one-dimensional case), but we have not been able to find the results we need explicitly stated and proved.

3.0.1. Bohr-Sommerfeld, Birkhoff normal form and resonant tori. The notion of resonant tori is based on the Bohr-Sommerfeld quantization rules CV2 for the joint spectrum of $Q_{1}, \ldots, Q_{n}$. In terms of microlocal analysis, these rules are encoded in the regular quantum Birkhoff 'inverse' normal form construction in Lemma (2.1). Fix a neighbourhood, $\Omega^{(l)}(b)$, of the torus $\Lambda^{(l)}(b)$ and let $\phi_{b, \hbar}$ be a microlocal solution of the system of equations:

$$
Q_{j} \phi_{b, \hbar}=\Omega_{\Omega^{(l)}}\left(b_{j}+\mu_{j}(\hbar)\right) \phi_{b, \hbar}+\mathcal{O}\left(\hbar^{\infty}\right), \text { with } \mu_{j}(\hbar)=o(1) ; j=1, \ldots, n .
$$

Here, by a slight abuse of notation, we have written $\mu_{j}$ in place of $\mu_{j k}$. In addition, suppose that

$$
\left\|\phi_{b, \hbar}\right\|_{\Omega^{(l)}} \geq C \hbar^{m}, \text { for some } m \in \mathbb{Z} .
$$

Then, from the 'inverse' Birkhoff normal form construction in Lemma 4.1, it follows that, for $j=1, \ldots, n$,

$$
\sum_{k=0}^{\infty} f_{j k}^{(l)}\left(\mu_{1}(\hbar), \ldots, \mu_{n}(\hbar)\right) \hbar^{k-1}=2 \pi \mathbb{Z}+\frac{\pi}{2}\left[\nu^{(l)}\right]+\mathcal{O}\left(\hbar^{\infty}\right)
$$

Let $\gamma_{j}^{(l)} ; j=1, \ldots, n$ denote the homology generators of the torus $\mathbb{T}^{n}$ and $\kappa$ denote the subprincipal form associated with $Q_{1}, \ldots, Q_{n}$ (see, for instance [GS2]). Then, to first approximation, the equation in (23) just the well-known formula:

$$
\frac{1}{\hbar} \int_{\gamma_{j}^{(l)}} \xi d x+\int_{\gamma_{j}^{(l)}} \kappa+\frac{\pi}{2}\left[\nu^{(l)}\right]=2 \pi \mathbb{Z}+\mathcal{O}\left(\hbar^{2}\right) .
$$

The asymptotic identities in (23) are called the Bohr-Sommerfeld (B-S) quantization rules for the joint eigenvalues $\left(b_{1}+\mu_{1}(\hbar), \ldots, b_{n}+\mu_{n}(\hbar)\right)$ of $\left(Q_{1}, \ldots, Q_{n}\right)$ associated with the component torus, $\Lambda^{(l)}\left(b_{1}, \ldots, b_{n}\right)$.

Note that in Lemma (2.1), we could have just as easily derived the Birkhoff normal form as:

$$
V^{-1} g\left(\hbar D_{\theta_{1}}, \ldots, \hbar D_{\theta_{n}} ; \hbar\right) V={ }_{\Omega^{(l)}} Q_{j}-b_{j},
$$


where, $g_{j}\left(x_{1}, \ldots, x_{n} ; \hbar\right) \sim \sum_{k} g_{j k}\left(x_{1}, \ldots, x_{n}\right) \hbar^{j}$, the $g_{j k}$ 's being the inverse functions of the $f_{j k}$ 's in Lemma (2.1) and $V: C^{\infty}\left(\Omega^{(l)}\right) \rightarrow C^{\infty}\left(\mathbb{R}^{n} \times \mathbb{S}^{1}\right)$ a microlocally unitary $\hbar$-Fourier integral operator. This way around, we get the (inverse) Bohr-Sommerfeld quantization rules expressing the $b_{1}+\mu_{1}, \ldots, b_{n}+\mu_{n}$ in terms of the quantum numbers, $m_{j}(\hbar) \in \hbar(\mathbb{Z}+\pi / 2[\nu])+$ $\mathcal{O}\left(\hbar^{\infty}\right)$ :

$$
\sum_{k=0}^{\infty} g_{j k}^{(l)}\left(m_{1}(\hbar), \ldots, m_{n}(\hbar)\right) \hbar^{k}=\mu_{j}(\hbar)+\mathcal{O}\left(\hbar^{\infty}\right) ; j=1, \ldots, n .
$$

In the following, we will need to consider two separate cases, roughly corresponding to whether or not the functions $f_{j k}^{(l)} ; l=1, \ldots, k$ (or equivalently, the $g_{j k}^{(l)} ; l=1, \ldots, k$ ) are all the same for the different component Lagrangian tori.

We can now define resonance between tori on the same level of the moment map. Let $b \in \mathbb{R}^{n}$ be a fixed regular value of the moment map $\mathcal{P}$ and consider the ladder of eigenvalues

$$
\Sigma_{b}(\hbar):=\left\{\left(b_{1}+\mu_{1}(\hbar), \ldots, b_{n}+\mu_{n}(\hbar)\right) \in \operatorname{Spec}\left(Q_{1}, \ldots, Q_{n}\right) ; \forall j=1, \ldots, n \mu_{j}(\hbar)=o(1)\right\} .
$$

We say that the component tori, $\Lambda^{(l)}\left(b_{1}, \ldots, b_{n}\right) ; l=1, \ldots, k$, are not in resonance if there exist eigenvalues $\left(\lambda_{1}, \ldots, \lambda_{n}\right) \in \Sigma_{b}(\hbar)$ which satisfy the Bohr-Sommerfeld (B-S) quantization rules (23) associated with the torus, $\Lambda^{(1)}\left(b_{1}, \ldots, b_{n}\right)$ to $\mathcal{O}\left(\hbar^{\infty}\right)$, but only solve the (B-S) rules associated with the other component tori, $\Lambda^{(l)}\left(b_{1}, \ldots, b_{n}\right)$, with $l \neq 1$ up to some finite order $m<\infty$. If a pair of tori on the same level satisfy precisely the same (B-S) quantization conditions, then they are said to be resonant.

3.1. Localization in the non-resonant case. The relationship between quasimodes and modes can be fairly complicated when there are resonances between component Lagrangian tori. However, as we now show, when the generic non-resonance condition is satisfied, the relationship is quite simple. In the following, we let $\mathcal{O}_{b}$ denote the usual order symbol $\mathcal{O}$ with locally regular dependence on $b \in B_{\text {reg }}$.

Lemma 3.1. Suppose $\phi_{b, \hbar}$ is an $L^{2}$-normalized joint eigenfunction of $Q_{1}, \ldots, Q_{n}$ with joint eigenvalues $\left(\lambda_{1}, \ldots, \lambda_{n}\right) \in \Sigma_{b}(\hbar) ; j=1, \ldots, n$ that satisfies the $(B-S)$ rules to infinite order only for the single component torus, $\Lambda^{(1)}\left(b_{1}, \ldots, b_{n}\right)$ (ie. the component tori, $\Lambda^{(l)}(b) ; l=1, \ldots, m$ are not in resonance). Then,

$$
\left(O p_{\hbar}\left(\chi^{(1)}\right) \phi_{b, \hbar}, O p_{\hbar}\left(\chi^{(1)}\right) \phi_{b, \hbar}\right)=1+\mathcal{O}_{b}\left(\hbar^{\infty}\right) .
$$

Here, $O p_{\hbar}\left(\chi^{(1)}\right)$ denotes the pseudodifferential cut-off with symbol $\chi^{(1)}(x, \xi)$ supported in $\Omega^{(1)}(b)$.

Proof: As we have already shown in Proposition (2.2), the space of microlocal solutions to (19) is at most one-dimensional. Suppose then that $\phi_{b, \hbar}$ is a joint eigenfunction of $Q_{1}, \ldots, Q_{n}$ with eigenvalues $\left(b_{1}+\mu_{1}, \ldots, b_{n}+\mu_{n}\right) \in \Sigma(\hbar)$. In particular, $\phi_{b, \hbar}$ is then a microlocal solution near each component torus; that is,

$$
Q_{j} \phi_{b, \hbar}=\Omega_{\Omega^{(l)}}\left(b_{j}+\mu_{j}(\hbar)\right) \phi_{b, \hbar}+\mathcal{O}_{b}\left(\hbar^{\infty}\right) .
$$


Consequently, given Proposition (2.2), there are two possibilities: In the first case, given $\chi^{(l)}(x, \xi) \in C_{0}^{\infty}\left(\Omega^{(l)}\right)$, a cutoff supported near the torus, $\Lambda^{(l)}$, we have that $\left(O p_{\hbar}\left(\chi^{(l)}\right) \phi_{b, \hbar}, O p_{\hbar}\left(\chi^{(l)}\right) \phi_{b, \hbar}\right) \geq$ $C \hbar^{m}$ for some $m \in \mathbb{Z}$. In such a situation, we already know that the eigenvalues $\left(b_{1}+\right.$ $\left.\mu_{1}, \ldots, b_{n}+\mu_{n}\right) \in \Sigma_{b}(\hbar)$ must satisfy the (B-S) quantization rules corresponding to the component torus, $\Lambda^{(l)}$, to $\mathcal{O}_{b}\left(\hbar^{\infty}\right)$. The only other possibility is that

$$
\left(O p_{\hbar}\left(\chi^{(l)}\right) \phi_{b, \hbar}, O p_{\hbar}\left(\chi^{(l)}\right) \phi_{b, \hbar}\right)=\mathcal{O}_{b}\left(\hbar^{\infty}\right) .
$$

Given the definition of non-resonance above, it follows that the latter scenario holds for $l \neq 1$ and the lemma follows.

As a direct consequence of Lemma 3.1, it follows that in the in non-resonant case, $L^{2}$ normalized quasimodes attached to individual Lagrangian tori agree with actual joint-eigenfunctions, modulo $\mathcal{O}\left(\hbar^{\infty}\right)$ errors in $L^{2}$. Moreover, we can now also prove the following correspondence principle for the case of $\mathbb{R}^{n}$ actions:

Proposition 3.2. Let $q(x, \xi) \in C_{0}^{\infty}\left(T^{*} M\right)$ and suppose that the component tori, $\Lambda^{(l)}(b) ; l=$ $1, \ldots, m$ are not in resonance. Then, for all $L^{2}$-normalized joint eigenfunctions, $\phi_{b, \hbar}$, with joint eigenvalues $\left(b_{1}+\mu_{1}(\hbar), \ldots, b_{n}+\mu_{n}(\hbar)\right) \in \Sigma_{b}(\hbar)$ with $\mu_{j}(\hbar)=\mathcal{O}(\hbar)$, there exists a component torus, $\Lambda^{(1)}(b)$, such that:

$$
\left(O p_{\hbar}(q) \phi_{b, \hbar}, \phi_{b, \hbar}\right)=(2 \pi)^{-n} \int_{\Lambda^{(1)}(b)} q d \mu_{b}+\mathcal{O}_{b}(\hbar) .
$$

Here, $d \mu_{b}$ denotes Liouville measure on $\Lambda^{(1)}(b)$.

Proof: The proof is similar to the case of torus actions [Z1]. For the sake of completeness, we sketch the argument. First, in light of Lemma (3.1),

$$
\left(O p_{\hbar}(q) \phi_{b, \hbar}, \phi_{b, \hbar}\right)=\left(O p_{\hbar}(q) \circ O p_{\hbar}\left(\chi^{(1)}\right) \phi_{b, \hbar} O p_{\hbar}\left(\chi^{(1)}\right) \phi_{b, \hbar}\right)+\mathcal{O}\left(\hbar^{\infty}\right) .
$$

A standard application of averaging, combined with the semiclassical Egorov theorem and the normal form in Lemma gives:

$$
\left(O p_{\hbar}(q) \chi^{(1)} \phi_{b, \hbar,} \chi^{(1)} \phi_{b, \hbar}\right)=(2 \pi)^{-n} \int_{\Lambda^{(1)}} q d \mu+e(\hbar)+\mathcal{O}(\hbar)
$$

where,

$$
e(\hbar)=\left(O p_{\hbar}(r) u_{\hbar}, u_{\hbar}\right)
$$

and $r \in C_{0}^{\infty}\left(\mathbb{T}^{n} \times D_{1}\right)$ with $r(\theta, I)=\mathcal{O}(|I|)$ and $u_{\hbar}(\theta)=\exp \left[i\left(m_{1} \theta_{1}+\ldots+m_{n} \theta_{n}\right)\right]$. An integration by parts in the $I_{1}, \ldots, I_{n}$ variables shows that:

$$
\left(O p_{\hbar}(r) u_{\hbar}, u_{\hbar}\right)=\mathcal{O}(\hbar) .
$$

The proposition follows.

3.2. Localization in the resonant case. In resonant cases, modes do not necessarily localize on individual tori. We introduce the language of 'quantum limit measures' to describe localization in more general cases. 
3.2.1. Quantum limits of modes and quasimodes. Let us denote by $M_{I}$ the set of measures on $S^{*} M$ which are invariant under the Hamiltonian $\mathbb{R}^{n}$-action. Among such measures are the orbital averaging measures $\mu_{z}$, defined by $\int_{S^{*} M} f d \mu_{z}=\lim _{T \rightarrow \infty} \frac{1}{T^{n}} \int_{\max \left|t_{j}\right| \leq T} f\left(\Phi^{t}(z)\right) d t$. In the case of compact (torus) orbits, one gets the normalized (probability) Lebesgue measure on the torus.

By the 'quantum limit' measures $\mathcal{Q}$ of the quantum integrable system, we mean the set of weak ${ }^{*}$ limits of the measures $d \Phi_{\lambda}$ defined by

$$
\left\langle O p(a) \phi_{\lambda}, \phi_{\lambda}\right\rangle=\int_{S^{*} M} a d \Phi_{\lambda},
$$

where $O p(a)$ is the pseudodifferential operator (in some fixed quantization) with complete symbol $a$. It is an easy consequence of Egorov's theorem that $\mathcal{Q} \subset M_{I}$. For background, terminology and references in a context closely related to this one, we refer to [TZ].

When $b$ is a regular value of the moment map $\mathcal{P}, \mathcal{P}^{-1}(b)$ is given by (20), where the $\Lambda^{(l)}(b) ; l=1, \ldots, m$ are $n$-dimensional Lagrangian tori. Let $d \mu_{\Lambda^{(j)}(b)}$ denote the normalize Lesbegue measures on such tori. If $\left\{\phi_{\lambda_{j}}\right\}$ is a sequence of eigenfunctions with $\left.\frac{\lambda_{j}}{\left|\lambda_{j}\right|}\right\} \rightarrow b$, then we have (see [T2])

$$
d \Phi_{\lambda_{j}} \rightarrow \sum_{j=1}^{m} c_{j} d \mu_{\Lambda^{(j)}(b)}, \quad \text { for some } \quad c_{j} \geq 0, \sum c_{j}=1 .
$$

It is difficult to determine the coefficients in general. As we have seen, in the generic ('nonresonant') case, one can find for each component torus a sequence of eigenfunctions for which $c_{j}=0, j \geq 2$.

Quite analogous to the quantum limit measures are the analogous measures defined for quasi-modes associated to regular tori. We thus define the set $\mathcal{Q}_{q m} \subset M_{I}$ of 'quasimode limit measures' to be the weak* limits of the analogous measures $d \Psi_{\lambda}$ corresponding to quasimodes $\left\{\psi_{\lambda}\right\}$ associated to regular tori. The following lemmas will be crucial for the proof of the Theorem in the $\mathbb{R}^{n}$ case:

LEMMA 3.3. For any component Lagrangian torus $\Lambda^{(l)}(b)$, there exists a sequence $\psi_{b, \hbar}^{(l)}$ of quasimodes such that

$$
\left(O p_{\hbar}(q) \psi_{b, \hbar}^{(l)}, \psi_{b, \hbar}^{(l)}\right)=(2 \pi)^{-n} \int_{\Lambda^{(l)}} q d \mu_{b}+\mathcal{O}_{b}(\hbar),
$$

i.e. that $\mathcal{Q}_{q m}$ contains all normalized Lebesgue measures along compact Lagrangean orbits.

Proof: Since the quasimodes $\psi_{b, \hbar}^{(l)}$ are, by construction, microlocally concentrated on a particular component torus, $\Lambda^{(l)}(b)$, Lemma (3.3) is proved in the same way as Proposition (3.2) above.

\section{Proof of Theorem (0.1)}

4.1. Pointwise bounds on quasimodes. In order to prove Theorems 0.1 in the possibly resonant case, we will need to establish some less direct relations between modes and quasimodes. The next Lemma shows that uniform bounded of sup-norms of modes implies the same for quasimodes: 
Lemma 4.1. Assume $\left\|\phi_{\lambda}\right\|_{\infty} \leq A$ for all $\lambda \in \operatorname{Spec}\left(P_{1}, \ldots, P_{n}\right)$. Then for all regular $b \in \mathbb{R}^{n}$, the $L^{2}$-normalized quasimodes satisfy:

$$
\limsup _{h \rightarrow 0}\left\|\psi_{b, h}^{(l)}\right\|_{\infty} \leq A m_{c l}(b) .
$$

\subsubsection{Proof of Lemma (4.1). Proof:}

Given the component tori $\Lambda^{(l)}(b)$ where $l=1, \ldots, m$, recall that we have denoted by $\psi_{b, \hbar}^{(l)}$ the quasimodes in Corollary (2.3) with approximate eigenvalues $\left(b_{1}+\mu_{1}, \ldots, b_{n}+\mu_{n}\right) \in \Sigma_{b}(\hbar)$ which are microlocally concentrated near the component torus, $\Lambda^{(l)}(b)$, . By making a Gram-Schmidt orthogonalization, we can assume that these functions are orthonormal in $L^{2}$. Recall, they satisfy for each $j=1, \ldots, n$ :

$$
Q_{j} \psi_{b, \hbar}^{(l)}=\left(b_{j}+\mu_{j}(\hbar)\right) \psi_{b, \hbar}^{(l)}+\mathcal{O}_{b}\left(\hbar^{\infty}\right)
$$

in $L^{2}$. Given the (B-S) rules, we know that for any component torus $\Lambda^{(l)}(b)$, there exist $\epsilon_{b}^{(l)}>0$ such that for $\hbar$ sufficiently small, the gaps in the (B-S) lattice spectrum are greater than $\epsilon_{b}^{(l)} \hbar$. Define

and consider the cube,

$$
\epsilon_{b}:=\min _{l \leq m_{c l}(b)} \epsilon_{b}^{(l)}
$$

$$
C_{b, \hbar}:=\prod_{j=1}^{n}\left[-\epsilon_{b} \hbar+b_{j}+\mu_{j}(\hbar), \epsilon_{b} \hbar+b_{j}+\mu_{j}(\hbar)\right] .
$$

Let $\mathbf{Q}:=\left(Q_{1}, \ldots, Q_{n}\right)$ act on $L^{2}(M) \oplus \cdots \oplus L^{2}(M)$ componentwise. Then, we denote by $\mathcal{Q}_{b, \hbar}^{(l)}$ the (at most) one-dimensional vector space generated by the quasimodes attached to $\Lambda^{(l)}(b)$ with quasieigenvalues in $C_{b, \hbar}$. In addition, we define

$$
\mathcal{Q}_{b, \hbar}:=\bigoplus_{l=1}^{m_{c l}(b)} \mathcal{Q}_{b, \hbar}^{(l)}
$$

So, put another way, $\mathcal{Q}_{b, \hbar}$ is the vector space of functions generated by quasimodes $\psi_{b, \hbar}^{(l)} ; l=$ $1, \ldots, m_{c l}$ from all of the component tori with quasieigenvalues in the cube $C_{b, \hbar}$. Similarily, we denote by $\mathcal{R}_{b, \hbar}$ the vector space generated by joint eigenfunctions of $\mathbf{Q}$ associated with joint eigenvalues of $\mathbf{Q}$ lying in $C_{b, \hbar}$. As a consequence of the (B-S) rules, we know that there are gaps in the joint spectrum of the $\mathrm{Q}$ : that is, there exists $C_{b}>0$ such that for all $\hbar \in\left(0, \hbar_{0}(b)\right]$

$$
\operatorname{dist}\left(\operatorname{Spec}(\mathbf{Q})-\operatorname{Spec}\left(\mathbf{Q} \cap C_{b, \hbar}\right), \operatorname{Spec}(\mathbf{Q})\right) \geq \frac{1}{C_{b}} \hbar .
$$

Let $E_{\mathcal{R}_{b, \hbar}}$ denote the spectral projector onto the eigenvalues of $\mathbf{Q}$ lying in $\mathcal{R}_{b, \hbar}$ and consider a fixed quasimode, $\psi_{b, \hbar} \in \mathcal{Q}_{b, \hbar}$. It follows that:

$$
\mathbf{Q}\left(\psi_{b, \hbar}-E_{\mathcal{R}_{b, \hbar}} \psi_{b, \hbar}\right)=\mathcal{O}_{b}\left(\hbar^{\infty}\right) .
$$

So, by the spectral theorem,

$$
\left\|\psi_{b, \hbar}-\sum_{\phi_{b, \hbar} \in R_{b, \hbar}}\left(\psi_{b, \hbar}, \phi_{b, \hbar}\right) \phi_{b, \hbar}\right\|_{(0)}=\mathcal{O}_{b}\left(\hbar^{\infty}\right) .
$$


Conversely, given $\phi_{b, \hbar} \in \mathcal{R}_{b, \hbar}$, it follows from the microlocal characterization result in Proposition (2.2) that:

$$
\left\|\phi_{b, \hbar}-\sum_{\psi_{b, \hbar} \in \mathcal{Q}_{b, \hbar}}\left(\phi_{b, \hbar}, \psi_{b, \hbar}\right) \psi_{b, \hbar}\right\|_{(0)}=\mathcal{O}_{b}\left(\hbar^{\infty}\right) .
$$

Let $d\left(\mathcal{Q}_{b, \hbar}, \mathcal{R}_{b, \hbar}\right)=\left\|\Pi_{\mathcal{Q}_{b, \hbar}}-\Pi_{\mathcal{Q}_{b, \hbar}} \Pi_{\mathcal{R}_{b, \hbar}}\right\|_{(0)}$ denote the non-symmetric distance between the vector spaces $\mathcal{Q}_{b, \hbar}$ and $\mathcal{R}_{b, \hbar}$. It follows from (32) and (33) that, in particular, $d\left(\mathcal{Q}_{b, \hbar}, \mathcal{R}_{b, \hbar}\right)<1$ and $d\left(\mathcal{R}_{b, \hbar}, \mathcal{Q}_{b, \hbar}\right)<1$. Consequently $[\mathrm{H}], \mathcal{Q}_{b, \hbar} \cong \mathcal{R}_{b, \hbar}$. Since $\mathcal{Q}_{b, \hbar}$ has dimension uniformly bounded independent of $\hbar$, so does $\mathcal{R}_{b, \hbar}$. In particular, we have that

$$
\operatorname{dim} \mathcal{R}_{b, \hbar} \leq m_{c l}(b) .
$$

Given the estimates in (31)-(34), we have by the Garding and Sobolev inequalities,

$$
\left\|\psi_{b, h}^{(l)}\right\|_{\infty} \leq\left\|E_{R_{b, h}} \psi_{b, h}^{(1)}\right\|_{\infty}+C\left\|(I-\Delta)^{s}\left(\psi_{b, \hbar}^{(l)}-E_{R_{b, h}} \psi_{b, h}^{(1)}\right)\right\|_{(0)} .
$$

The lemma follows from the following two estimates:

$$
\begin{aligned}
& \left\|E_{R_{b, h}} \psi_{b, h}^{(1)}\right\|_{\infty}=\left\|\sum_{j=1}^{m(b)}\left\langle\psi_{b, h}^{(1)}, \phi_{b, h}^{(j)}\right\rangle \phi_{b, h}^{(j)}\right\|_{\infty} \leq A m_{c l}(b) \\
& \left\|(I-\Delta)^{s}\left(\psi_{b, \hbar}^{(l)}-E_{R_{b, h}} \psi_{b, h}^{(1)}\right)\right\|_{(0)}=\mathcal{O}_{b}\left(\hbar^{\infty}\right)
\end{aligned}
$$

4.2. Completion of the proof. The completion of the proof is now exactly as in [TZ], so we only briefly recall it. We first observe:

LEMma 4.2. Let $\left\{\pi_{*} d \mu_{\Lambda}\right\}$ denote the set of projections to $M$ of normalized Lebesgue measures on compact Lagrangean tori $\Lambda \subset S^{*} M$. Then the family is uniformly bounded as linear functionals on $L^{1}(M)$.

Proof: By Lemma (3.3), each $d \mu_{\Lambda}$ is a weak limit of quasi-mode measures corresponding to a sequence, say $\left\{\psi_{\Lambda, h}\right\}$, of quasimodes. By Lemma (4.1) we have, for $V \in C(M)$,

$$
\begin{aligned}
& \left|\int_{M} V \pi_{*} d \mu_{\Lambda}\right|=\lim _{h \rightarrow 0}\left|\left\langle V \psi_{\Lambda, h}, \psi_{\Lambda, h}\right\rangle\right| \leq\|V\|_{L^{1}} \lim \sup _{h \rightarrow 0}\left\|\psi_{\Lambda, h}\right\|_{\infty}^{2} \\
& \leq A^{2} m_{c l}(\mathcal{P}(\Lambda))^{2}\|V\|_{L^{1}} .
\end{aligned}
$$

COROLlary 4.3. The invariant Lagrangean tori project without singularities to the base $M$.

Proof: Indeed, we have $\pi_{*} d \mu_{\Lambda}=f_{\lambda} d v o l$ for some $f_{\lambda} \in L^{\infty}(M)$. But it is straightforward to see that if $\left.\pi\right|_{\Lambda}$ has singularities, then $f_{\lambda}$ will blow up along them.

The geometric problem then arises, which completely integrable systems have the property that all invariant tori project without singularities to the base $M$ ? It follows immediately that $M$ must be a torus, and that no leaf of the Liouville foliation is singular. By a theorem of Mane, it then follows that the metric has no conjugate points. But the only metrics on a torus without conjugate points are the flat ones (Burago-Ivanov). For more details, we refer to [TZ]. 


\section{EXAMPlES OF QUANTUM COMPLETELY INTEGRABLE SYSTEMS}

To illustrate some of the variety of quantum completely integrable systems, we describe the Liouville foliations of two infinite dimensional families of two-dimensional examples: surfaces of revolution and Liouville tori.

5.1. Spheres of revolution. Suppose that $g$ is a metric on $S^{2}$ for which there exists an effective action of $S^{1}$ by isometries of $\left(S^{2}, g\right)$. The two fixed points will be denoted $N, S$ and $(r, \theta)$ will denote geodesic polar coordinates centered at $N$, with $\theta=0$ some fixed meridian $\gamma_{M}$ from $N$ to $S$. The metric may then be written in the form $g=d r^{2}+a(r)^{2} d \theta^{2}$ where $a:[0, L] \rightarrow \mathbb{R}^{+}$is defined by $a(r)=\frac{1}{2 \pi}\left|S_{r}(N)\right|$, with $\left|S_{r}(N)\right|$ the length of the distance circle of radius $r$ centered at $N$. For any smooth surface of revolution, the function $a$ satisfies $a^{(2 p)}(0)=a^{(2 p)}(L)=0, a^{\prime}(0)=1, a^{\prime}(L)=-1$ and two such surfaces $M_{1}, M_{2}$ are isometric if and only if $L_{1}=L_{2}$ and $a_{1}(r)=a_{2}(r)$ or $a_{1}(r)=a_{2}(L-r)$.

Complete integrability of $H=|\xi|_{g}$ (i.e. of the geodesic flow) is classical, and follows from the existence of the Clairaut integral $p_{\theta}(v):=\left\langle v, \frac{\partial}{\partial \theta}\right\rangle$. Since the Poisson bracket $\left\{p_{\theta},|\xi|_{g}\right\}=$ 0 , the geodesics are constrained to lie on the level sets of $p_{\theta}$; and since both $|\xi|_{g}$ and $p_{\theta}$ are homogeneous of degree one, the behaviour of the geodesic flow is determined by its restriction to $S_{g}^{*} S^{2}=\left\{|\xi|_{g}=1\right\}$. The moment map is given by

$$
P=\left(|\xi|_{g}, p_{\theta}\right): T^{*} S^{2} \rightarrow \mathbb{R}^{2}
$$

To describe the inverse images, i.e. the invariant tori, we need to specify the function $a(r)$. We will assume it is a Morse function on $[0, L]$ with critical points at $\left\{m_{k}\right\} \subset(0, L)$.

5.1.1. Case 1: generic case. For simplicity and with no real loss of generality, let us assume that there are two local maxima $M_{1}, M_{2}$ with $a\left(M_{2}\right)=C_{2}>a\left(M_{1}\right)=C_{1}$ and one local minimum $m_{2}$ with $a\left(m_{2}\right)=c_{2}$. We note that the canonical involution $(x, \xi) \rightarrow(x,-\xi)$ preserves $\mathcal{P}^{-1}\left(p_{1}, p_{2}\right)$, and that only the meridian torus $\mathcal{P}^{-1}\left(p_{1}, 0\right)$ is invariant; otherwise there are always at least two components on each level. By homogeneity it suffices to consider the level sets $\mathcal{P}^{-1}(1, c)$ in the unit tangent bundle. We will denote the components by $M_{c}^{( \pm, k)}$ where \pm denotes some ordering of each pair of tori interchanged by the involution. The following table summarizes the invariant tori, their projections to $M$ and the type of singularity of the projection.

$\begin{array}{llll}\text { Range of } c & \text { components } M_{c}^{( \pm, k)} & \text { projections to } M & \text { singularities } \\ \text { (0) } c>C_{2} \text { or } c<0 & \text { none } & - & - \\ \text { (a) } c=C_{2} & 2 \text { circles } & \text { Project to closed geodesics } & \text { singular leaf } \\ \text { (b) } C_{1}<c<C_{2} & 2 \text { torii } & 2 \text { horizontal annuli } & \text { fold } \\ \text { (c) } c_{1}<c<C_{1} & 4 \text { tori } & \text { each projects diffeo to } M & \text { none } \\ \text { (d) } c=c_{1} & 4 \text { cylinders } & \text { each projects over all of } M & \text { none } \\ \text { (e) } 0<c<c_{1} & 2 \text { tori } & \text { projects to } 2 \text { horizontal annuli } & \text { fold } \\ \text { (f) } c=0 & 1 \text { (meridian) torus } & \text { covers all of } M & \text { blow-down. }\end{array}$


5.1.2. Simple surfaces of revolution. Simple surfaces of revolution are examples of toric integrable systems [CV1] They are the special surfaces where:

(i) $a$ has precisely one non-degenerate critical point $r_{o} \in(0, L)$, with $a^{\prime \prime}\left(r_{o}\right)<0$, corresponding to an 'equatorial geodesic' $\gamma_{E}$;

(i) the (non-linear) Poincare map $\mathcal{P}_{\gamma_{E}}$ for $\gamma_{E}$ is of twist type (cf. $\S 1$ ).

It is proved in CV1 that the geodesic flow of a simple surface of revolution is is toric integrable.

With the extra assumption on $a$, the level sets are compact and the only critical level is that of the equatorial geodesics $\gamma_{E}^{ \pm} \subset S_{g}^{*} S^{2}$ (traversed with either orientation). The other level sets consist of two-dimensional torii.

As above, let

$$
P=\left(|\xi|_{g}, p_{\theta}\right): T^{*} S^{2} \rightarrow B:=\left\{\left(b_{1}, b_{2}\right):\left|b_{2}\right| \leq a\left(r_{o}\right) b_{1}\right\} \subset \mathbb{R} \times \mathbb{R}^{+}
$$

be the moment map of the Hamiltonian $\mathbb{R}^{2}$-action defined by the geodesic flow and by rotation. The singular set of $P$ is the closed conic set $Z:=\left\{\left(r_{o}, \theta, 0, p_{\theta}\right): \theta \in[0,2 \pi), p_{\theta} \in \mathbb{R}\right\}$, i.e. $Z$ is the cone thru the equatorial geodesic (in either orientation). The image of $Z$ is the boundary of $B$; the map $\left.P\right|_{T^{*} S_{g} S^{2}-Z}$ is a trivial $S^{1} \times S^{1}$ bundle over the open convex cone $B_{o}$ (the interior of $B$ ). For each $b \in B_{o}$, let $H_{1}\left(F_{b}, \mathbb{Z}\right)$ denote the homology of the fiber $F_{b}:=P^{-1}(b)$. This lattice bundle is trivial since $B$ is contractible, so there exists a smoothly varying homology basis $\left\{\gamma_{1}(b), \gamma_{2}(b)\right\} \in H_{1}\left(F_{b}, \mathbb{Z}\right)$ which equals the unit cocircle $S_{N}^{*} S^{2}$ together with the fixed closed meridian $\gamma_{M}$ when $b$ is on the center line $\mathbb{R}^{+} \cdot(1,0)$. The action variables are given by [CV1], $\S 6$,

$$
I_{1}(b)=\int_{\gamma_{1}(b)} \xi d x=p_{\theta}, \quad I_{2}(b)=\int_{\gamma_{2}(b)} \xi d x=\frac{1}{\pi} \int_{r_{-}(b)}^{r_{+}(b)} \sqrt{b_{1}^{2}-\frac{b_{2}^{2}}{a(r)^{2}}} d r+\left|b_{2}\right|
$$

where $r_{ \pm}(b)$ are the extremal values of $r$ on the annulus $\pi\left(F_{b}\right)$ (with $\pi: S^{*} S^{2} \rightarrow S^{2}$ the standard projection). On the torus of meridians in $S^{*} S^{2}$, the value of $I_{2}$ equals $\frac{L}{\pi}$ and it equals one on the equatorial geodesic. So extended, $I_{1}, I_{2}$ are smooth homogeneous functions of degree 1 on $T^{*} S^{2}$, and generate $2 \pi$-periodic Hamilton flows. It follows that the pair $\mathcal{I}:=\left(I_{1}, I_{2}\right)$ generates a global Hamiltonian torus $\left(S^{1} \times S^{1}\right)$-action commuting with the geodesic flow. We refer to it as the 'standard' Hamiltonian $T^{2}$ action on $T^{*} S^{2}-0$. Up to symplectic equivalence, it is unique.

The singular set of $\mathcal{I}$ equals $\mathcal{Z}:=\left\{I_{2}= \pm p_{\theta}\right\}$, corresponding to the equatorial geodesics. The map

$$
\mathcal{I}: T^{*} S^{2}-\mathcal{Z} \rightarrow \Gamma_{o}:=\left\{(x, y) \in \mathbb{R} \times \mathbb{R}^{+}:|x|<y\right\}
$$

is a trivial torus fibration. Henceforth we often write $T_{I}$ for the torus $\mathcal{I}^{-1}(I)$ with $I \in \Gamma_{o}$ and let $\Gamma=\mathcal{C} \downarrow \Gamma_{o}$ be the closure of $\Gamma$ as a convex cone. The symplectically dual angle variables $\left(\phi_{1}=\theta, \phi_{2}\right)$ then give, by definition, the flow times $(\bmod 2 \pi)$ along the orbits of $\left(I_{1}, I_{2}\right)$ from a fixed point on $F_{b}$, which we may take to be the unique point lying above the intersection 
of the equator and the fixed meridian on $F_{b}$ with the geodesic pointing into the northern hemisphere.

The following table summarizes the invariant tori and their projections

$\begin{array}{llll}\text { Range of } c & \text { components } M_{c}^{( \pm, k)} & \text { projections to } M & \text { singularity } \\ \text { (0) } c>C \text { or } c<0 & \text { none } & - & - \\ \text { (a) } c=C & 2 \text { circles } & \text { Project to closed geodesics } & \text { singular leaf } \\ \text { (b) } 0<c<C & 2 \text { torii } & 2 \text { horizontal annuli } & \text { fold } \\ \text { (c) } c=0 & 1 \text { (meridian) torus } & \text { covers all of } M & \text { blow-down. }\end{array}$

5.2. Tori of revolution. Here, we consider a metric $g$ on $T^{2}$ which possesses a free effective action $R_{\theta}$ of $S^{1}$ by isometries. We denote the generator of this action by $Z$. Associated to $Z$ is the Clairaut integral $p_{Z}: T^{*} T^{2} \rightarrow \mathbb{R}$ defined by $p_{Z}(\nu)=\langle\nu, Z\rangle$. The $S^{1}$ action on $T^{2}$ lifts to $T^{*}\left(T^{2}\right)$ as the Hamiltonian flow $\phi^{t}$ of $p_{\theta}$. Since $\left\{|\xi|_{g}, p_{Z}\right\}=0$, we have a Hamiltonian $G=\mathbb{R}^{1} \times S^{1}$ action $\Phi^{t}=G^{t} \times R_{\theta}$ on $T^{*}\left(T^{2}\right)$ with moment map $\mathcal{P}=\left(|\xi|_{g}, p_{Z}\right)$.

Consider now the level set

$$
M=\mathcal{P}^{-1}(1,0)=\left\{(x, \xi):|\xi|_{g}=1,\langle\xi, \partial / \partial \theta\rangle=0\right\}
$$

It is an affine Lagrangean submanifold of $T^{*}\left(T^{2}\right)$ and since it is compact it must be a Lagrangean torus. It follows that the stabilizer $G_{m}$ of a point of $m \in M$ must be a subgroup of the form $L \mathbb{Z} \times\{i d\}$. In particular, the $G^{t}$-orbit of each $m \in M$ must be closed.

As above, let us restrict the natural projection $\pi: S_{g}^{*} T^{2} \rightarrow T^{2}$ to $M$, to get $\pi_{M}: M \rightarrow M$. Clearly, $\pi_{M}$ is equivariant with respect to the $S^{1}$ action $R_{\theta}$ on $T^{2}$ and its lift. It sends the $G^{t}$-orbits of $m \in M$ to closed geodesics of $T^{2}$ and since $p_{Z}=0$ on $M$, these geodesics are orthogonal to the $R_{\theta}$-orbits, which we will call 'parallels'. Further, we note that the tangent space $T_{m} M$ is spanned by the Hamilton vector fields $\Xi_{|\xi|_{g}}$, resp. $\Xi_{p_{Z}}$. Since $\pi_{M *}$ sends these vectors to orthogonal vectors, $\pi_{M}$ is a submersion and hence a covering map. Its degree is one because of the equivariance and the fact that it sends $G^{t}$-orbits to closed geodesics (this also follows from Lalonde's theorem cited above). Hence it is a diffeomorphism. We will refer to the geodesics in $M$ as the meridians.

Fixing a point $m_{o} \in M$ and $x_{o}=\pi_{M}\left(m_{o}\right) \in T^{2}$ we get a coordinate system on $T^{2}$ with coordinates $(r, \theta): x=(r, \theta) \Leftrightarrow \Phi^{(r, \theta)}\left(m_{o}\right)=\pi_{M}^{-1}(x)$. Since the meridians are orthogonal to the 'parallels', this forms an orthogonal coordinate system for $g$. Hence $g$ has the form: $g=d r^{2}+a(r)^{2} d \theta^{2}$.

5.2.1. Liouville torii [B.K.S [KMS]. A Riemannian torus $(M, g)$ of dimension 2 is called a Liouville torus if the metric has the form

$$
g=\left[U_{1}\left(x_{1}\right)-U_{2}\left(x_{2}\right)\right]\left(d x_{1}^{2}+d x_{2}^{2}\right)
$$

in the coordinates $\left(x_{1}, x_{2}\right)$ on $[0,1] \times[0,1]$. The functions $U_{j}$ are assumed to be periodic of period 1 and to satisfy $U_{1}\left(x_{2}\right)-U_{2}\left(x_{2}\right)>0$. The geodesic flow is the Hamilton flow on $T^{*} M$ 
generated by

$$
H(x, \xi)^{2}=\frac{1}{\left[U_{1}\left(x_{1}\right)-U_{2}\left(x_{2}\right)\right.}\left(\xi_{1}^{2}+\xi_{2}^{2}\right) .
$$

It Poisson commutes with the first integral

$$
S(x, \xi)=\frac{U_{2}\left(x_{2}\right)}{\left[U_{1}\left(x_{1}\right)-U_{2}\left(x_{2}\right)\right.} \xi_{1}^{2}+\frac{U_{1}\left(x_{1}\right)}{\left[U_{1}\left(x_{1}\right)-U_{2}\left(x_{2}\right)\right.} \xi_{2}^{2} .
$$

We then have the homogeneous moment map

$$
\mathcal{P}=\left(p_{1}, p_{2}\right)=\left(H, \frac{S}{H}\right): T^{*} M-0 \rightarrow \mathbb{R}^{2} .
$$

We note that the canonical involution $(x, \xi) \rightarrow(x,-\xi)$ preserves $\mathcal{P}^{-1}\left(p_{1}, p_{2}\right)$, and that no torus is invariant; hence there are always at least two components on each level. As above, it suffices to consider the level sets in the unit tangent bundle, which we continue to denote by $M_{c}^{( \pm, k)}$.

To describe the inverse images, i.e. the invariant tori, we need to specify the functions $U_{j}\left(x_{j}\right)$. We will assume that $U_{j}$ is a Morse function on $[0,1]$ with critical points at $\left\{c_{j k}\right\} \subset$ $(0,1)$ with critical values $\left\{v_{j k}\right\}$. We briefly review the description of the tori under the assumption that each $U_{j}$ has precisely one maximum $c_{j+}$ and one minimum $c_{j-}$ and that the critical points do not coincide (see [KMS for a detailed description). We thus have $c_{1+}>c_{11}>c_{2+}>c_{2-}$.

$\begin{array}{llll}\text { Range of } c & \text { components } M_{c}^{( \pm, k)} & \text { projections to } M & \text { singularity } \\ \text { (0) } c>c_{1+} \text { or } c<c_{2-} & \text { none } & - & - \\ \text { (a) } c=c_{1+} & 2 \text { circles } & \text { Project to closed geodesics } & \text { singular leaf } \\ \text { (b) } c_{1-}<c<c_{1+} & 2 \text { torii } & 2 \text { horizontal annuli } & \text { folds } \\ \text { (c) } c=c_{1-} & 4 \text { cylinders } & \text { each projects over all of } M & \text { none } \\ \text { (d) } c_{2+}<c<c_{1-} & 4 \text { tori } & \text { each projects diffeo to } M & \text { none } \\ \text { (a') } c=c_{2+} & \text { analogous to (a) } & 2 \text { circles } & \text { singular leaves } \\ \text { (b') } c_{2-}<c<c_{2+} & \text { analogous to (b) } & 2 \text { vertical annuli } & \text { folds } \\ \text { (c') } c=c_{2-} & \text { analogous to (c) } & \end{array}$

5.2.2. Tori of revolution: Quantum theory. Consider the metric on $\mathbb{R}^{2} / \mathbb{Z}^{2}$

$$
g=a(x)\left(d x^{2}+d \xi^{2}\right)
$$

where $a$ is a periodic function. Translations in the $\xi$ variable are then isometries so this is a torus of revolution. The Laplacian

$$
\Delta=\frac{1}{a} \Delta_{0}
$$

commutes with $\partial / \partial \xi$ and hence there is an ONB of joint eigenfunctions of the form

$$
\phi_{N, \lambda}=e^{2 \pi i N \xi} \psi_{N, \lambda}(x) \text {. }
$$

The eigenvalue problem is separable in these variables and gives

$$
\psi^{\prime \prime}-4 \pi^{2} N^{2} \psi=-\lambda a \psi
$$


Bourgain [Bour] observes that for $a=1-\tau \sin ^{2} \pi x$ and for sufficiently large $N$ there are eigenfunctions satisfying

$$
\left\|\phi_{\lambda}\right\|_{\infty} \geq \lambda^{\frac{1}{8}}, \quad\left\|\phi_{\lambda}\right\|_{L^{2}}=1
$$

These eigenfunctions concentrate near the projection of a stable geodesic given by the parallel $x=0$. Note that Theorem 0.2 gives in this case a lower bound of $\left\|\phi_{\lambda}\right\|_{\infty} \geq C(\epsilon) \lambda^{\frac{1}{8}-\epsilon}$ for any $\epsilon>0$. Since $d a(x)=-2 \pi \sin \pi x \cos \pi x$ and since $x \in[0,1]$ there are two critical circles: at $x=0$ and at $x=\frac{1}{2}$, a maximal parallel and a minimal parallel (which are geodesics). There are four embedded cylinders consisting of geodesics which spiral in towards the latter circle.

5.2.3. Liouville tori: Quantum theory B.K.S [KMS]. The Laplace Beltrami operator on a Liouville torus, $Q$, with metric $g=\left[U_{1}\left(x_{1}\right)-U_{2}\left(x_{2}\right)\right]\left(d x_{1}^{2}+d x_{2}^{2}\right)$ is just:

$$
\Delta=\left[U_{1}\left(x_{1}\right)-U_{2}\left(x_{2}\right)\right]^{-1}\left(\frac{\partial^{2}}{\partial x_{1}^{2}}+\frac{\partial^{2}}{\partial x_{2}^{2}}\right) .
$$

The commuting operator with principal symbol $S(x, \xi)$ in 5.4 is just:

$$
S=\left[U_{1}\left(x_{1}\right)-U_{2}\left(x_{2}\right)\right]^{-1}\left(U_{2}\left(x_{2}\right) \frac{\partial^{2}}{\partial x_{1}^{2}}+U_{1}\left(x_{1}\right) \frac{\partial^{2}}{\partial x_{2}^{2}}\right) .
$$

The joint eigenfunctions of $\Delta$ and $S$ can be described by two coupled O.D.E.(see K.M.S.). Moreover, in the case where $c=c_{1+}$ or $c=c_{2+}$ the two circles in the joint level set are stable and elliptic and project to a single geodesic on $Q$. In the case $c=c_{1+}$ this projected geodesic is identified with the line given by $x_{1}=M_{1}$ where $U_{1}\left(x_{1}\right)$ attains its non-degenerate maximum at this point, whereas, when $c=c_{2+}$ this geodesic gets identified with the line $x_{2}=M_{2}$ where $U_{2}\left(x_{2}\right)$ attains its maximum at $M_{2}$. There are eigenfunctions attached to these levels which satisfy $\left\|\phi_{\lambda}\right\|_{\infty} \geq \lambda^{\frac{1}{8}}$ and again this is consistent with the prediction given by Theorem 0.2 . Note also that there exist hyperbolic geodesics on the level sets given by $c=c_{1-}$ and $c=c_{2-}$ which are limit sets for the geodesics on these cylinders. These are the lines given by $x_{1}=m_{1}$ and $x_{2}=m_{2}$ respectively, where $m_{1}$ and $m_{2}$ are the points where $U_{1}$ and $U_{2}$ achieve their respective minima. In this case, [T1], one can show that there are joint eigenfunctions concentrating near the projections of these hyperbolic orbits and satisfy $\left\|\phi_{\lambda}\right\|_{\infty} \geq \lambda^{\frac{1}{8}}(\log \lambda)^{-\frac{1}{2}}$, slightly better than the $\left\|\phi_{\lambda}\right\|_{\infty} \geq C(\epsilon) \lambda^{\frac{1}{8}-\epsilon}$ given by Theorem 0.2 .

5.3. Other examples. Other classical examples include the Euler top and geodesic on asymmetric ellipsoids in any dimension. These examples are also quantum completely integrable. Tri-axial ellipsoids have special umbilic points where all directions are geodesic loop directions. Hence they are candidates for maximal eigenfunction growth. The loops form a Lagrangean manifold with boundary which has a blow-down projection over the umbilic points. We do not believe however that the eigenfunctions actually do have maximal sup norm growth. 


\section{FURTHER DISCUSSION AND OPEN PROBLEMS}

In [TZ], we list a number of (still) open problems in this area. We would like to mention three of them here since they seem rather glaringly open.

- Are the results on quantum completely integrable systems valid under the weaker assumption of classical complete integrability? E.g. if $\Delta$ is a quantum integrable Laplacian, and if its eigenfunctions have a given growth rate, can the additition of a lower order term such as $\Delta+V$ with $V \in C^{\infty}(M)$ change the growth rate?

- Is the existence of a stable elliptic closed geodesic sufficient for the existence of a sequence $\left\{\phi_{\lambda}\right\}$ of eigenfunctions satisfying $\left\|\phi_{\lambda}\right\|_{\infty} \sim \lambda^{\frac{n-1}{8}}$ ? Note that if would be sufficient to obtain sup norm estimates for KAM systems.

- How do the results generalize to Schroedinger operators $\Delta+V$ on $\mathbb{R}^{n}$ ? Here, we may assume $V$ grows at infinity to ensure that the spectrum is discrete.

\section{REFERENCES}

[AA] O. Agam and B. L. Altshuler, "Scars" in parametrically excited surface waves, xxx.lanl.gov condmat/0004190).

[A] V. I. Arnold, Modes and quasimodes. Functional Analysis and its Applications 6 (1972), no. 2, $12-20$.

[ABST] Aurich, R.; Bcker, A.; Schubert, R.; Taglieber, M. Maximum norms of chaotic quantum eigenstates and random waves. Phys. D 129 (1999), no. 1-2, 1-14.

[AS] Aurich, R.; Steiner, F. Statistical properties of highly excited quantum eigenstates of a strongly chaotic system. Phys. D 64 (1993), no. 1-3, 185-214.

[Be2] M. V. Berry, Semi-classical mechanics in phase space: a study of Wigner's function. Philos. Trans. Roy. Soc. London Ser. A 287 (1977), 237-271.

[B.K.S] P.Bleher, D.Kosygin and Ya.G.Sinai, Distribution of energy levels of a quantum free particle on a Liouville surface and trace formulae, Comm.Math.Phys. 179 (1995), 375-403.

[BGS] Galya Blum, Sven Gnutzmann, and Uzy Smilansky, Nodal domains statistics - a criterion for quantum chaos, xxx.lanl.gov (nlin.CD/0109029).

[BS] E. Bogomolny and C. Schmit, Percolation model for nodal domains of chaotic wave functions, xxx.lanl.gov (nlin.CD/0110019).

[Bour] J.Bourgain, Eigenfunction bounds for compact manifolds with completely integrable geodesic flow, IHES preprint (1993).

[Ch] A.M. Charbonnel, Comportement semi-classique du spectre conjoint d'operateurs pseudodifferentiel qui commutent, Asympt. Anal. 1 (1988), 227-261.

[CHENG] S. Y. Cheng, Eigenfunctions and nodal sets. Comment. Math. Helv. 51 (1976), no. 1, 43-55.

[CP] Y.Colin de Verdiere and B.Parisse, Equilibre instable en regime semi-classique I: concentration microlocale, Comm. in P.D.E. 19 (1994), 1535-1563.

[CV1] Y.Colin de Verdiere, Spectre conjoint d'operateurs pseudodifferentiels qui commutent II: Le cas integrable, Math.Zeit. 171 (1980), 51-75.

[CV2] Y.Colin de Verdiere, Quasi-modes sur les varietes Riemanniennes compactes, Invent.Math. 43 (1977), 15-52.

[CV3] Y. Colin de Verdire, Sur une hypothse de transversalit d'Arnold, Comment. Math. Helv. 63 (1988), no. 2, 184-193.

[D] H. Donnelly, Bounds for eigenfunctions of the Laplacian on compact Riemannian manifolds (preprint, 2001).

[DF] H. Donnelly and C. Fefferman, Nodal sets of eigenfunctions on Riemannian manifolds. Invent. Math. 93 (1988), no. 1, 161-183. 
[DF2] H. Donnelly and C. Fefferman, Growth and geometry of eigenfunctions of the Laplacian. Analysis and partial differential equations, 635-655, Lecture Notes in Pure and Appl. Math., 122, Dekker, New York, 1990.

[E] K. Efetov, Supersymmetry in disorder and chaos. Cambridge University Press, Cambridge, 1997.

[FE] Falko, Vladimir I.; Efetov, K. B. Statistics of wave functions in mesoscopic systems. J. Math. Phys. 37 (1996), no. 10, 4935-4967.

[FHHMLE] G. F. Fiete, J. S. Hersch, E. J. Heller, H. C. Manoharan, C.P. Lutz, D. M. Eigler, Scattering Theory of Kondo Mirages and Observation of Single Kondo Atom Phase Shift (xxx.lanl.gov condmat/0008170)

[H] B.Helffer, Semiclassical analysis for the Schrodinger operator and applications, Lecture Notes vol. 1336, Springer-Verlag (1988).

[HS] Helffer, B.; Sjstrand, J. Multiple wells in the semiclassical limit. III. Interaction through nonresonant wells. Math. Nachr. 124 (1985), 263-313.

[HSu] J. L. van Hemmen and A. St, Semiclassical quantization and resonance in spin tunnelling. (English. English summary) J. Phys. A 31 (1998), no. 49, 10029-10043.

[IS] Iwaniec, H.; Sarnak, P. $L^{\infty}$ norms of eigenfunctions of arithmetic surfaces. Ann. of Math. (2) 141 (1995), no. 2, 301-320.

[JN] Jakobson, Dmitry; Nadirashvili, Nikolai Eigenfunctions with few critical points. J. Differential Geom. 53 (1999), no. 1, 177-182.

[JNT] D. Jakobson, N. Nadirashvili and J. Toth, Geometry of eigenfunctions (Russian Math. Surveys, to appear).

[JL] Jerison, David; Lebeau, Gilles Nodal sets of sums of eigenfunctions. Harmonic analysis and partial differential equations (Chicago, IL, 1996), 223-239, Chicago Lectures in Math., Univ. Chicago Press, Chicago, IL, 1999.

[K] Kaplan, L. Scars in quantum chaotic wavefunctions. Nonlinearity 12 (1999), no. 2, R1-R40.

[KH] Kaplan, L.; Heller, E. J. Weak quantum ergodicity. Phys. D 121 (1998), no. 1-2, 1-18.

[KMS] D. Kosygin, A. Minasov, Ya. G. Sină, Statistical properties of the spectra of Laplace-Beltrami operators on Liouville surfaces. (Russian) Uspekhi Mat. Nauk 48 (1993), no. 4(292), 3-130; translation in Russian Math. Surveys 48 (1993), no. 4, 1-142.

[L] Lazutkin, Vladimir F. KAM theory and semiclassical approximations to eigenfunctions. With an addendum by A. I. Shnirelman. Ergebnisse der Mathematik und ihrer Grenzgebiete (3) [Results in Mathematics and Related Areas (3)], 24. Springer-Verlag, Berlin, 1993.

[M] Martinez, Andr Precise exponential estimates in adiabatic theory. J. Math. Phys. 35 (1994), no. 8, 3889-3915.

[Mi] Mirlin, A. D. Statistics of energy levels and eigenfunctions in disordered and chaotic systems: supersymmetry approach. New directions in quantum chaos (Villa Monastero, 1999), 223-298, Proc. Internat. School Phys. Enrico Fermi, 143, IOS, Amsterdam, 2000.

[Mi2] Mirlin, Alexander D. Statistics of energy levels and eigenfunctions in disordered systems. Phys. Rep. 326 (2000), no. 5-6, 259-382.

[P] Popov, G. Invariant tori, effective stability, and quasimodes with exponentially small error terms. I. Birkhoff normal forms. Ann. Henri Poincar 1 (2000), no. 2, 223-248.

[R] Ralston, J. V. On the construction of quasimodes associated with stable periodic orbits. Comm. Math. Phys. 51 (1976), no. 3, 219-242.

[RS] Rudnick, Zev; Sarnak, Peter The behaviour of eigenstates of arithmetic hyperbolic manifolds. Comm. Math. Phys. 161 (1994), no. 1, 195-213.

[ShZ] B. Shiffman and S. Zelditch, Random spherical harmonics (in preparation).

[Sj] J.Sjostrand, Semi-excited states in nondegenerate potential wells, Asymp.Anal. 6(1992), 29-43.

[So1] C.D. Sogge, Concerning the $L^{p}$ norm of spectral clusters for second-order elliptic operators on compact manifolds. J. Funct. Anal. 77 (1988), no. 1, 123-138.

[So2] Sogge, Christopher D. Oscillatory integrals and spherical harmonics. Duke Math. J. 53 (1986), no. $1,43-65$. 
[SZ] C. D. Sogge and S. Zelditch, Riemannian manifolds with maximal sup norm growth, Duke Math J. (to appear.)

[T1] J.A.Toth, Eigenfunction localization in the quantized rigid body, J.Diff.Geom. 43(4)(1996), 844-858.

[T2] J.A.Toth, On the quantum expected values of integrable metric forms, J.Diff.Geom. (1998)(to appear).

[TZ] J. A. Toth and S. Zelditch, Riemannian manifolds with uniformly bounded eigenfunctions (to appear in Duke Math Journal).

[TZ2] J. A. Toth and S. Zelditch, $L^{p}$ norms of eigenfunctions in the completely integrable case, (to appear).

[Wi] M. Wilkinson, Tunnelling between tori in phase space. Phys. D 21 (1986), no. 2-3, 341-354.

[W] Wolpert, Scott A. The modulus of continuity for $\Gamma_{0}(m) \backslash \mathbb{H}$ semi-classical limits. Comm. Math. Phys. 216 (2001), no. 2, 313-323.

[Z] S. Zelditch, Appendix to: "Weak quantum ergodicity" [Phys. D 121 (1998), no. 1-2, 1-18; MR 99k:81071] by L. Kaplan and E. J. Heller. (English. English summary) Phys. D 121 (1998), no. 1-2, $19-23$.

[Z1] S.Zelditch, Quantum transition amplitudes for classically ergodic or completely integrable systems, J. Fun. Anal. 94 (1990), 415-436.

Department of Mathematics and Statistics, McGill University, Montreal, CANAdA, H3A-2K6

Department of Mathematics, Johns Hopkins University, Baltimore, MD 21218, USA 